\title{
Recipient mucosal-associated invariant T cells control GVHD within the colon
}

\author{
Antiopi Varelias, ${ }^{1,2}$ Mark D. Bunting, ${ }^{1}$ Kate L. Ormerod, ${ }^{3}$ Motoko Koyama, ${ }^{1}$ Stuart D. Olver, ${ }^{1}$ Jasmin Straube, ${ }^{4}$ Rachel D. Kuns, ${ }^{1}$ \\ Renee J. Robb, ${ }^{1}$ Andrea S. Henden, ${ }^{1,5}$ Leanne Cooper, ${ }^{4}$ Nancy Lachner, ${ }^{3}$ Kate H. Gartlan, ${ }^{1,2}$ Olivier Lantz, ${ }^{6}$ Lars Kjer-Nielsen, ${ }^{7}$ \\ Jeffrey Y.W. Mak, ${ }^{8}$ David P. Fairlie, ${ }^{8}$ Andrew D. Clouston, ${ }^{9}$ James McCluskey, ${ }^{7}$ Jamie Rossjohn, ${ }^{10,11,12}$ Steven W. Lane, ${ }^{2,4,5}$ \\ Philip Hugenholtz, ${ }^{3}$ and Geoffrey R. Hill',2,5

\begin{abstract}
'Bone Marrow Transplantation Laboratory, QIMR Berghofer Medical Research Institute, Brisbane, Australia. ${ }^{2}$ Faculty of Medicine, and ${ }^{3}$ Australian Centre for Ecogenomics, The University of Queensland, Brisbane, Australia. " ${ }^{4}$ ordon and Jessie Gilmour Leukaemia Research Laboratory, QIMR Berghofer Medical Research Institute, Brisbane, Australia. ${ }^{5}$ The Royal Brisbane and Women's Hospital, Brisbane, Australia. IINSERM U932 and Department de Biologie des Tumeurs, Institute Curie and Centre d'Investigation Clinique, CICBT507 ICR/Curie, Paris, France. 'Department of Microbiology and Immunology, Peter Doherty Institute for Infection and Immunity, University of Melbourne, Parkville, Australia. ${ }^{8}$ Australian Research Council Centre of Excellence in Advanced Molecular Imaging, Institute for Molecular Bioscience, The University of Queensland, Brisbane, Australia. ${ }^{9}$ Envoi Specialist Pathologists, Brisbane, Australia. ${ }^{10}$ nfection and Immunity Program and The Department of Biochemistry and Molecular Biology, Biomedicine Discovery Institute Monash University, Clayton, Australia. "Institute of Infection and Immunity, Cardiff University School of Medicine, Cardiff, United Kingdom. ${ }^{12}$ Australian Research
\end{abstract} \\ Council Centre of Excellence in Advanced Molecular Imaging, Monash University, Clayton, Australia.
}

\begin{abstract}
Mucosal-associated invariant T (MAIT) cells are a unique innate-like T cell subset that responds to a wide array of bacteria and yeast through recognition of riboflavin metabolites presented by the MHC class I-like molecule MR1. Here, we demonstrate using MR1 tetramers that recipient MAIT cells are present in small but definable numbers in graft-versus-host disease (CVHD) target organs and protect from acute GVHD in the colon following bone marrow transplantation (BMT). Consistent with their preferential juxtaposition to microbial signals in the colon, recipient MAIT cells generate large amounts of IL-17A, promote gastrointestinal tract integrity, and limit the donor alloantigen presentation that in turn drives donor Th1 and Th17 expansion specifically in the colon after BMT. Allogeneic BMT recipients deficient in IL-17A also develop accelerated CVHD, suggesting MAIT cells likely regulate GVHD, at least in part, by the generation of this cytokine. Indeed, analysis of stool microbiota and colon tissue from IL-17A-/- and $\mathrm{MR}^{-/-}$mice identified analogous shifts in microbiome operational taxonomic units (OTU) and mediators of barrier integrity that appear to represent pathways controlled by similar, IL-17A-dependent mechanisms. Thus, MAIT cells act to control barrier function to attenuate pathogenic T cell responses in the colon and, given their very high frequency in humans, likely represent an important population in clinical BMT.
\end{abstract}

\section{Introduction}

Acute graft-versus-host disease (GVHD) remains a major complication of allogeneic bone marrow transplantation (BMT) and results in considerable morbidity and mortality. Major target organs include the gastrointestinal (GI) tract, liver, and skin, all of which represent tissues with extensive environmental interfaces (1). Acute GVHD is initiated during conditioning when inflammation invoked by chemoradiotherapy serves to enhance antigen-presenting cell (APC) function and the costimulation of T cells in tissue (1). Recipient alloantigens are thus presented to donor $\mathrm{T}$ cells that differentiate along proinflammatory lineages defined by Th1 and Th17 paradigms (2). Target organ damage is mediated by both inflammatory cytokines generated by $\mathrm{T}$ cells and mononuclear cells together with $\mathrm{T}$ cell

Authorship note: $\mathrm{AV}$ and MDB contributed equally to this work

Conflict of interest: LKN, JR, JM, and DPF are named inventors on a patent application (PCT/AU2013/000742, WO2014005194). LKN, JYWM, JR, JM, and DPF are named inventors on a patent application (PCT/AU2015/050148, W02015149130) involving MR1 ligands for MR1-restricted MAIT cells owned by University of Queensland, Monash University, and University of Melbourne.

Submitted: November 7, 2016; Accepted: February 16, 2018.

Reference information: J Clin Invest. 2018;128(5):1919-1936.

https://doi.org/10.1172/JCI91646. cytotoxic pathways. Danger-associated molecular pattern (DAMP) and pathogen-associated molecular pattern (PAMP) signaling byproducts of cellular damage induced by chemoradiotherapy and microbiome-derived products are critical in driving this system-wide inflammation $(2,3)$.

The primacy of the GI tract in controlling the severity of GVHD is well established (4), and it is increasingly clear that, as the epithelial barrier loses integrity, microbiota-derived PAMPs induce the release of IL-17 and IL-22, among other cytokines, whose primary function is to limit pathogen spread. IL-17 in the GI tract is produced by conventional T cells, $\gamma \delta$ T cells, and the recently described mucosal-associated invariant $\mathrm{T}$ (MAIT) cells (5-7), the latter of which are exquisitely positioned to respond to microbial invasion by nature of their residence within the lamina propria of the small and large intestine (6-9). MAIT cells typically express semiinvariant $\mathrm{T}$ cell receptor (TCR) repertoires consisting of TRAV1 (Vo19 in mice or $\mathrm{V} \alpha 7.2$ in humans) joined to J $\alpha 33$ (also J $\alpha 12$ and $\mathrm{J} \alpha 20$ in humans) and limited TCR $\beta$ chain pairing (19 and 13 in mice, 6 and 20 in humans) (10). These innate-like $\mathrm{T}$ cells respond to vitamin B2- and B9-derived metabolites presented by the MHC class I-like molecule MR1 to rapidly secrete effector cytokines (11-15). The riboflavin-based precursors are produced by a range of bacteria, 
A

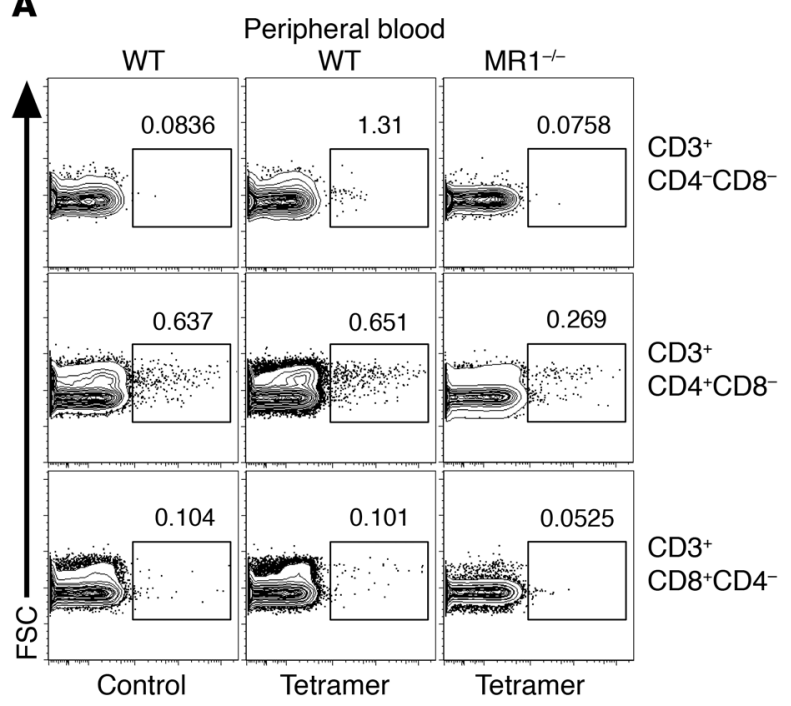

C

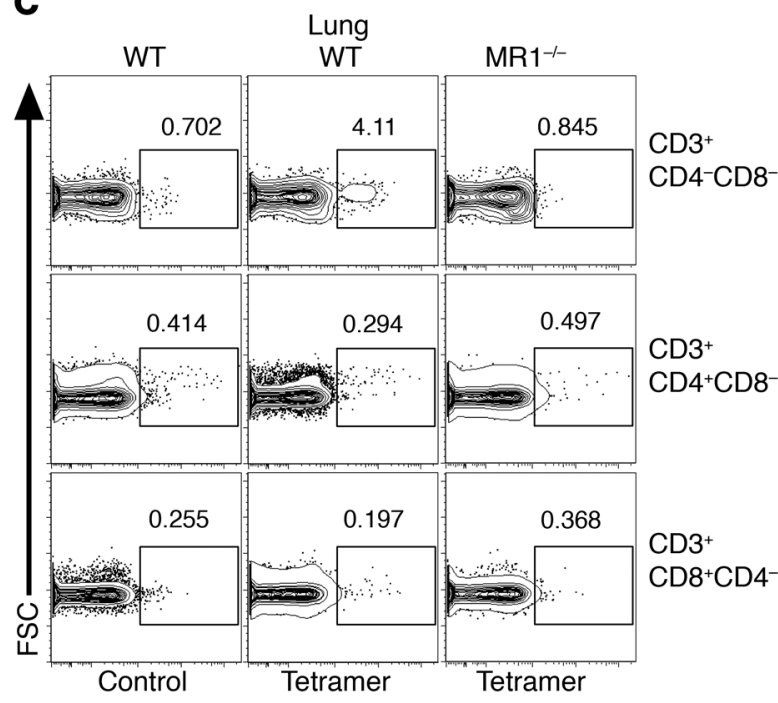

E

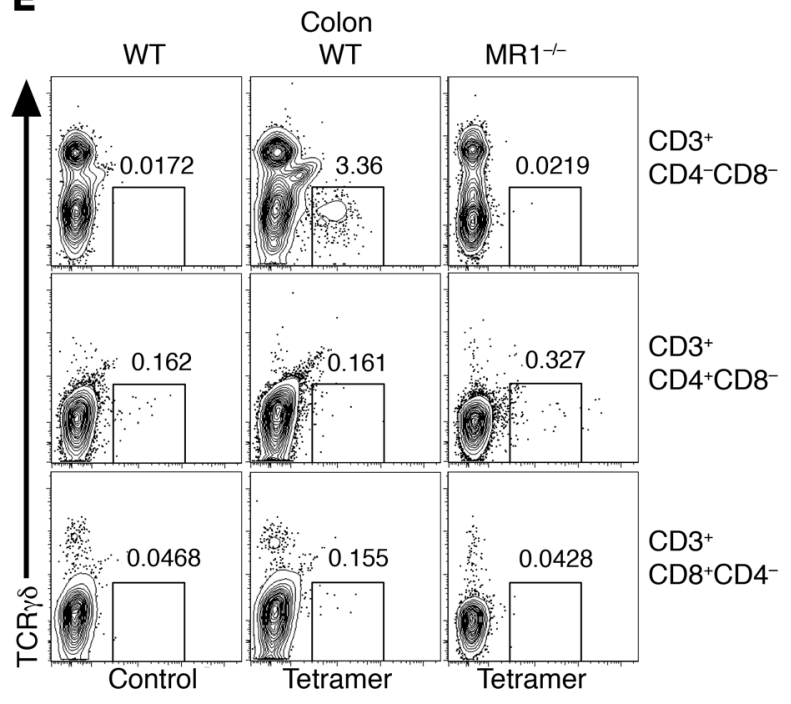

B

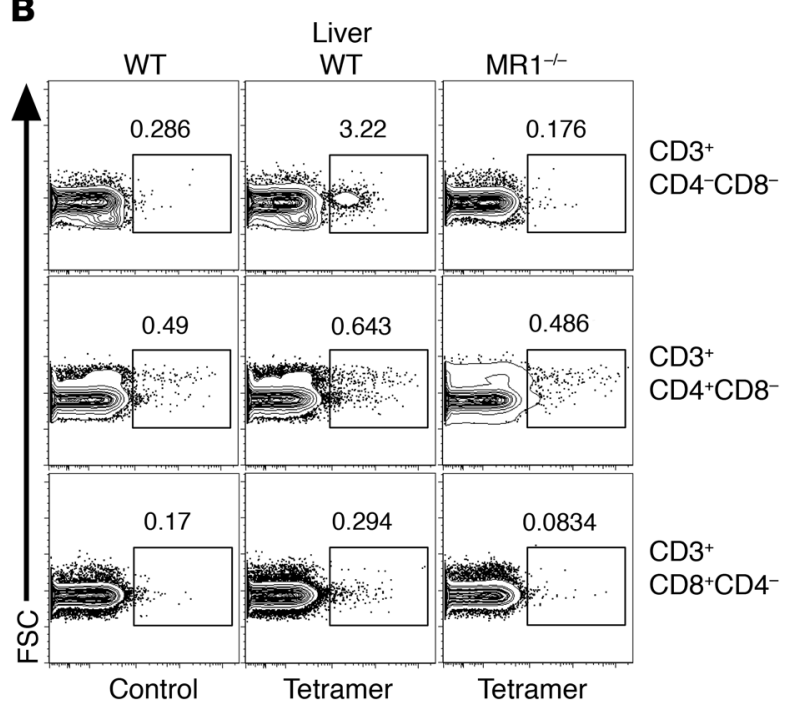

D

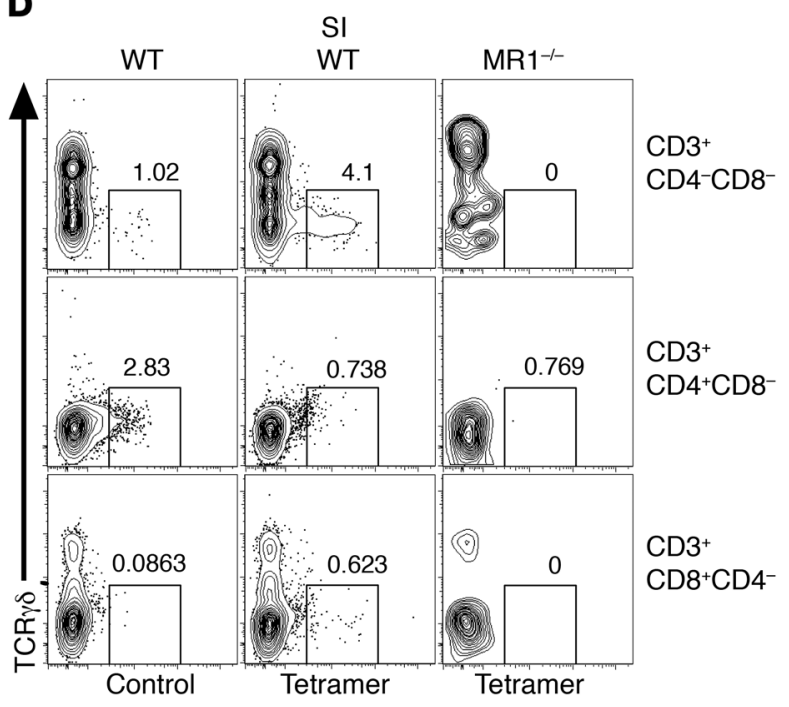

Figure 1. MAIT cells are present in GVHD target organs. Lymphocytes from naive mouse peripheral blood (A), liver (B), lung (C), SI (D), and colon (E) were stained with the MR1 tetramer (5-OP-RU) or control tetramer (6-FP) and analyzed by flow cytometry. Gates were set based on the control tetramer and cells from MR1 ${ }^{-1-}$ mice. Mouse MAIT cells were defined as CD3+. Representative dot plots are shown. 

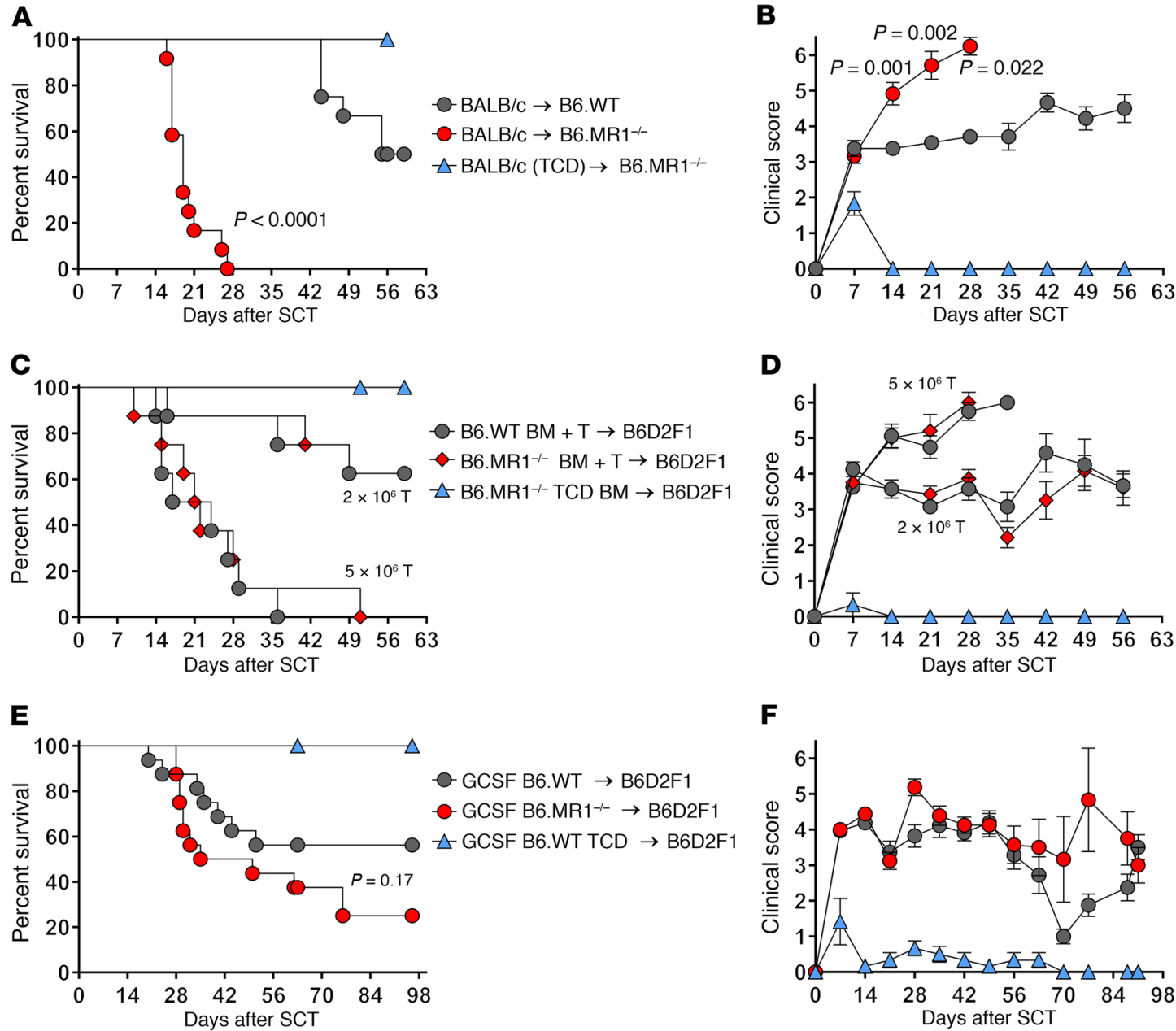

Figure 2. Recipient MAIT cells provide protection from GVHD. (A and B) G-CSF-mobilized BALB/c.WT splenocytes $\left(25 \times 10^{6}\right)$ were transplanted to lethally irradiated B6.WT or B6.MR1 ${ }^{-1-}$ mice and survival and clinical scores monitored. Data pooled from 2 independent experiments. $n=12$ per group; TCD group, $n=3$. (C and D) Grafts composed of B6.WT BM $\left(5 \times 10^{6}\right)$ and B6.WT T cells $\left(2\right.$ or $5 \times 10^{6}$ as indicated) or B6.MR1 $1^{-1-}$ BM and B6.MR1 ${ }^{-1-}$ T cells were transplanted into lethally irradiated B6D2F1 recipients and survival and clinical scores determined. Data combined from 2 independent experiments are shown. $n=16$ per group; TCD group, $n=7$. (E and F) Lethally irradiated B6D2F1 recipients were transplanted with G-CSF-mobilized splenocytes $\left(10 \times 10^{6}\right)$ from B6.WT or B6.MR1 ${ }^{-1-}$ donors. Data combined from 2 replicate experiments are shown. $n=16$ per group; TCD group, $n=6$ mice. Survival represented by Kaplan-Meier analysis.

yeast, and fungi (and not mammalian cells), such as Escherichia coli, Staphylococcus aureus, Mycobacterium tuberculosis, Salmonella typhimurium, Pseudomonas aeruginosa, Klebsiella pneumoniae, Lactobacillus acidophilus, and Candida albicans, to mention a few (16). This unique activation pathway provides an additional mechanism of determining nonself from self. Previous work in murine models and humans has shown that MAIT cells possess potent antimicrobial functions, primarily due to the rapid, diverse, and expansive cytokine production by these cells $(6,16-22)$. However, a role for MAIT cells in transplantation outcomes has not been reported to date. We hypothesized that MAIT cells would be intricately involved in regulating GVHD, as they are located at mucosal sites where GVHD manifests. We thus have utilized MAIT celldeficient $\mathrm{MR}^{-/-}$mice and MR1 tetramers $(7,8,11)$ to characterize the role of MAIT cells in the context of BMT.

\section{Results}

MAIT cells are present in GVHD target organs. MAIT cells were traditionally identified in adult humans using the surface markers
Va7.2 and CD161, where they are found in relatively high frequencies in the blood, liver, and GI tract $(8,23)$. However, identification of mouse MAIT cells using readily available surface markers is not feasible due to an absence of reagents and a specific surface-marker phenotype to distinguish these cells from conventional T cells. Using mouse MR1 tetramers loaded with either an activating MAIT cell ligand (5-[2-oxopropylideneamino]6-D-ribitylaminouracil [5-OP-RU]) or a nonactivating ligand (6-formylpterin [6-FP]) $(7,11)$ we were able to screen and specifically identify mouse MAIT cells by flow cytometry in various organs from naive mice. Lymphocytes from the peripheral blood, liver, lungs, small intestine (SI), and colon of naive mice were stained with the common T cell markers CD3, CD4, and CD8 and the MR1 tetramers described herein. MR1 $1^{-/}$mice, which lack MAIT cells, were used as an additional control to show specificity of the MR1 tetramer. MAIT cells were mostly $\mathrm{CD} 3^{+} \mathrm{CD} 4^{\text {neg }} \mathrm{CD} 8^{\text {neg }}$, with much smaller numbers of $\mathrm{CD}^{+} \mathrm{CD}^{+}$and $\mathrm{CD}^{+}{ }^{+} \mathrm{CD} 8^{+}$MAIT cells, and this varied among the tissues examined, consistent with recent studies (6-8). Compared to peripheral blood (Figure 1A), a higher frequen- 
A $\square$ BALB/C.WT $\rightarrow$ B6.WT

$\square \mathrm{BALB} / \mathrm{c} . \mathrm{WT} \rightarrow \mathrm{B} 6 . \mathrm{MR} 1^{-/-}$
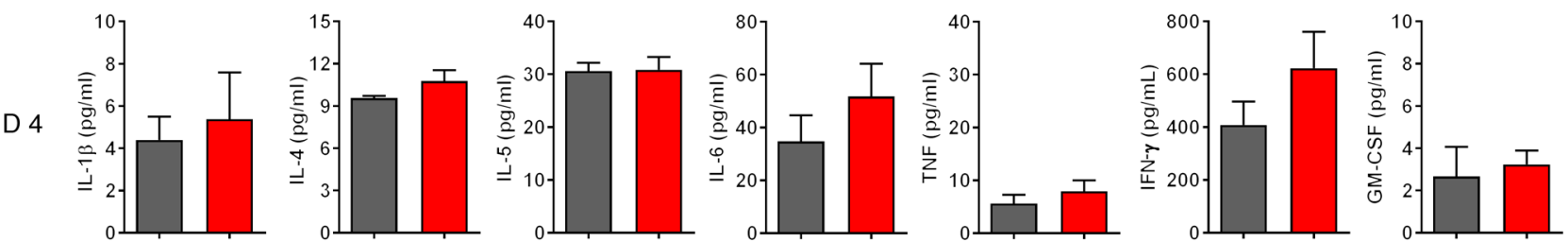

$\mathrm{D} 7$ 豙 $6-1$
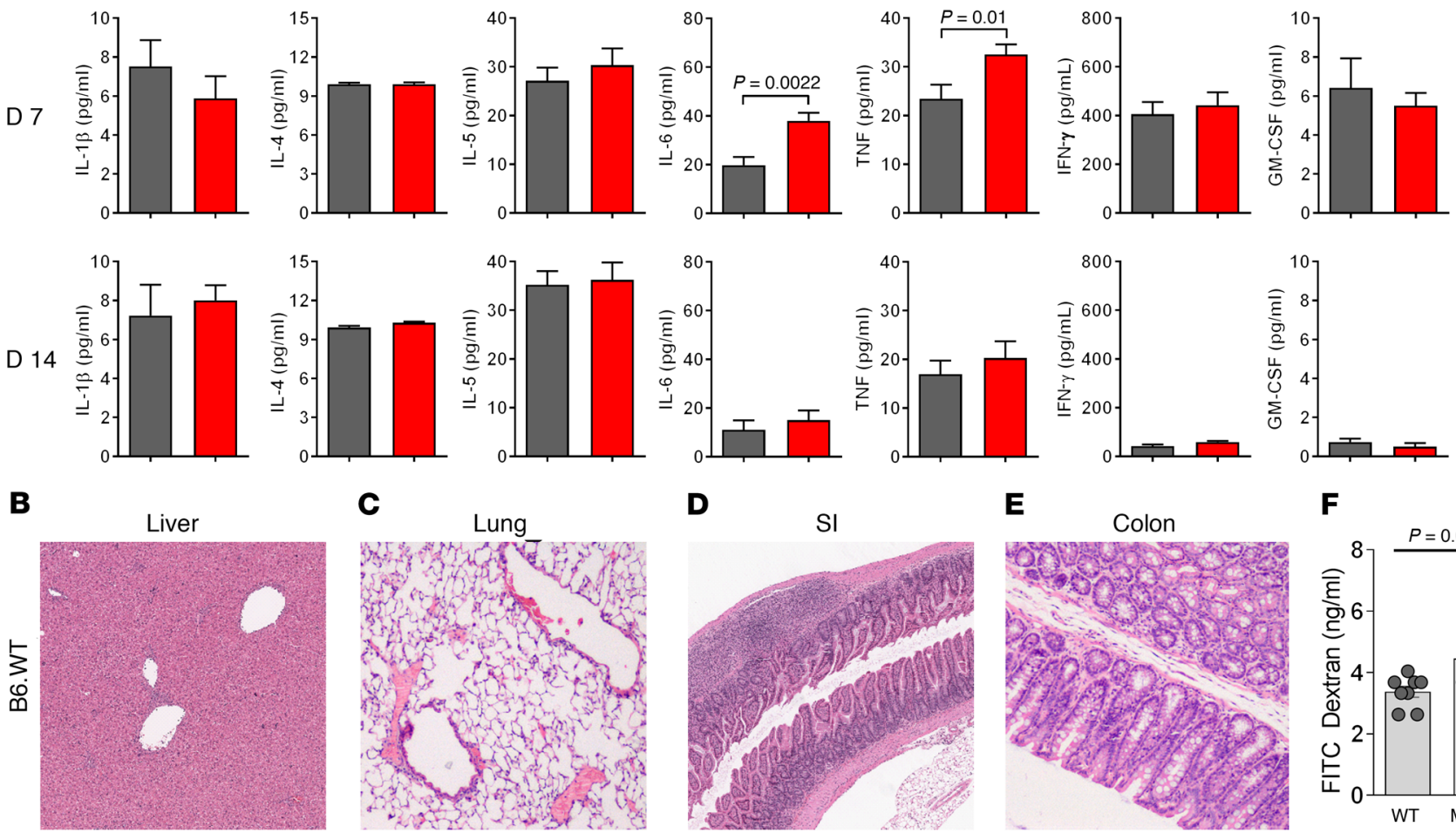

C

D

E Colon

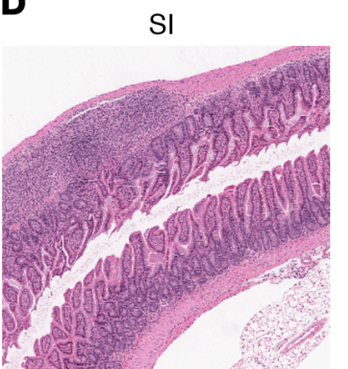

$\mathbf{F}$
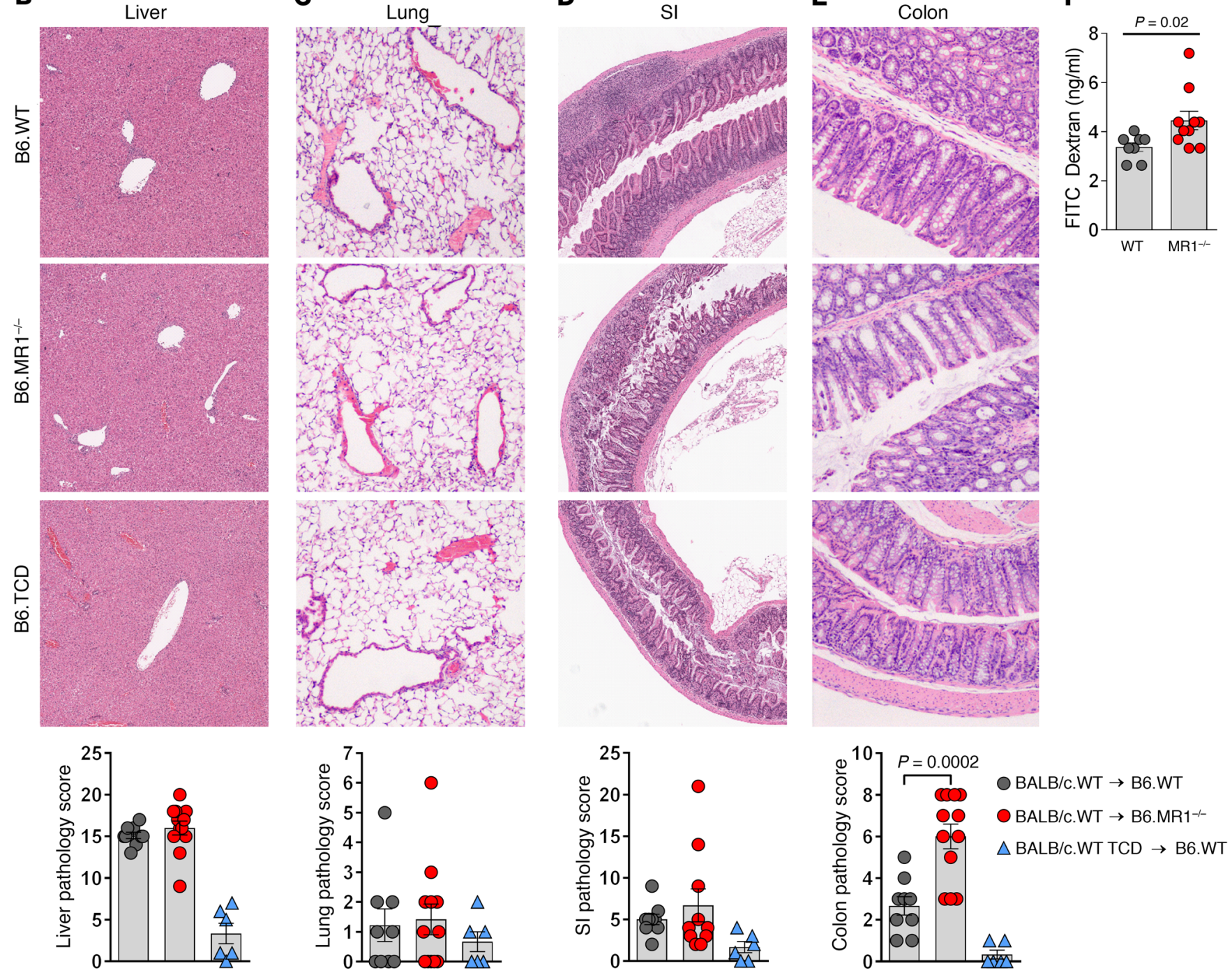
Figure 3. Recipient MAIT cells attenuate acute GVHD within the GI tract. B6.WT and B6.MR1 ${ }^{-/-}$mice were transplanted with G-CSF-mobilized BALB/c.WT splenocytes or TCD splenocytes. (A) Serum cytokine analysis was conducted on days 4, 7, and 14 after transplant. Day 4 data from 1-2 experiments. $n=5-10$ per group; day 7 data pooled from 2 independent experiments, $n=11-12$ per group; day 14 data from 1 experiment. $n=6-7$ per group. (B-E) Semiquantitative histopathology of liver (B), lung (C), SI (D), and colon (E) from B6.WT and B6.MR1 ${ }^{-1-}$ recipients at days $13-14$ after SCT. Images were captured on a Nikon ECLIPSE Ci microscope fitted with a DS-Fi2 camera. Original magnification, $\times 4$. Data combined from 2 independent experiments, $n=9-12$ per group; TCD group, $n=6$. (F) Perturbed intestinal barrier integrity as determined by FITC-dextran levels in serum on day 13 after SCT. All data analyzed using the Mann-Whitney $U$ test except histology data, which was analyzed using the unpaired $t$ test with Welch's correction.

cy of MAIT cells was noted in the liver (Figure 1B), lung (Figure 1C), SI, and colon (Figure 1, D and E), approximating $3 \%-4 \%$ of gated $\mathrm{CD}^{+} \mathrm{CD} 4^{\text {neg }} \mathrm{CD} 8^{\text {neg }}$ cells, as defined by binding of the active MR1 tetramer. Fractionation of the SI and colon tissue into intraepithelial and lamina propria compartments revealed that MAIT cells resided exclusively within the lamina propria (data not shown). Additionally, detection of MR1 tetramer-positive cells in the spleen, lymph nodes (LNs), and bone marrow was negligible (data not shown), highlighting the mucosal-associated nature of mouse MAIT cells. These data support those recently published using MR1 tetramers $(6,7)$ and suggest that MAIT cells may have a role to play in GVHD due to their localization in GVHD target organs and juxtaposition to potential environmental stimuli.

Recipient MAIT cells abrogate GVHD induced by allogeneic stem cell transplantation. Prior study of the function of MAIT cells has shown both proinflammatory and regulatory functions, depend- ing on the disease model used (24-28). We investigated the function of MAIT cells in a major MHC-mismatched allogeneic transplant setting. C57BL/6 WT (B6.WT) or MR1-deficient mice on a B6 background (B6.MR1 ${ }^{-/}$) were lethally irradiated and transplanted with granulocyte colony stimulating factor-mobilized (G-CSF-mobilized) BALB/c spleen grafts (stem cell transplant [SCT]). G-CSF-mobilized T cell-depleted (TCD) BALB/c splenocytes were also included as a non-GVHD control, and survival and clinical scores were assessed. B6.WT and B6.MR1/-- recipients showed a median survival time of 56 days and 19 days, respectively, corresponding to a significant reduction in survival (Figure 2A). Clinical scores of $\mathrm{B} 6 . \mathrm{MR}^{-1}$ - recipients were also significantly higher from day 14 to day 28 after transplant (Figure 2B). GVHD lethality was also increased in B6.MR1/- relative to B6.WT recipients that received grafts from $\mathrm{LP} / \mathrm{J}$ donors that were mismatched for minor histocompatibility antigens only (day 70 survival, 33\% versus $100 \% ; P=0.02$ ). These data indicate that recipient MAIT cells function in a regulatory manner in the setting of GVHD. To determine whether donor-derived MAIT cells contributed to regulation of GVHD, B6D2F1 mice were lethally irradiated and transplanted with either B6.WT or B6.MR1 ${ }^{--}$BM and T cells in a major MHC-mismatched model. Notably, naive $\mathrm{B} 6 . \mathrm{MR1}^{-/}$animals exhibited no perturbation of the conventional $\mathrm{T}$ cell compartment with respect to abundance and subsets (Supplemental Figure 1, A and B; supplemental material available online with this article; https://doi.org/10.1172/JCI91646DS1), suggesting that any effect on survival was due to the absence of MAIT cells alone. Survival and clinical scores were similar between B6.WT and B6.MR1 $1^{--}$donor grafts (Figure 2, C and D). We also performed transplants in a second system using G-CSF-mobilized donor grafts from $\mathrm{B} 6 . \mathrm{WT}$ and $\mathrm{B} 6 . \mathrm{MR}^{1 /-}$ mice into $\mathrm{B} 6 \mathrm{D} 2 \mathrm{~F} 1$ recip-
A
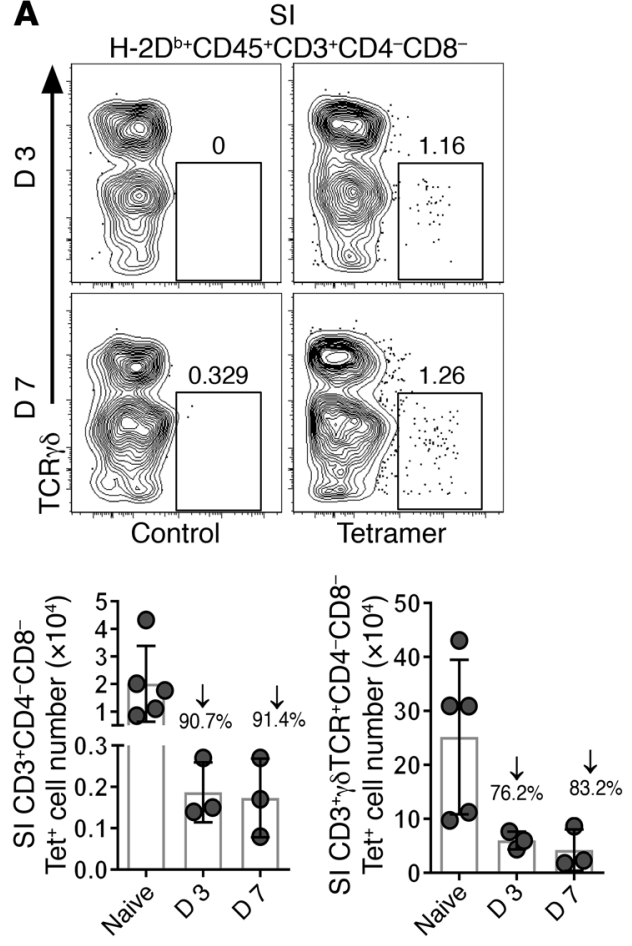

B
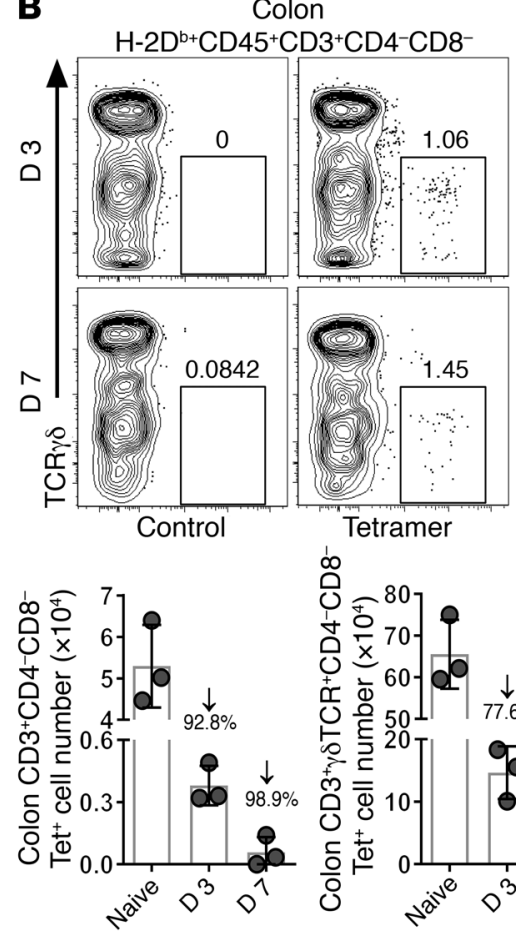

$\stackrel{\infty}{0}$

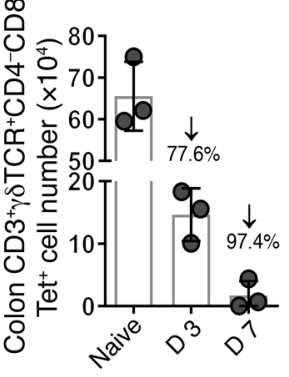

Figure 4. Recipient MAIT cells within the GI tract persist after BMT. B6.WT mice were lethally irradiated and transplanted with $10 \times 10^{6} \mathrm{BALB} / \mathrm{C} \mathrm{BM}$ and $3 \times 10^{6}$ BALB/c T cells. Recipient lamina propria lymphocytes from the SI (A) and colon (B) isolated at day 3 and day 7 after transplant were stained with control and MR1 tetramer and analyzed by flow cytometry. Representative dot plots of host CD45 ${ }^{+}$ $\mathrm{CD}^{+} \mathrm{CD} 4^{\text {nes }} \mathrm{CD} 8^{\text {neg }}$ cells additionally stained with $\gamma \delta$ TCR and MR1 tetramer are shown. Quantification of MAIT cell numbers before and after transplant for the $\mathrm{SI}$ and colon is shown below plots. Enumeration of recipient $\gamma \delta$ T cells from the same samples is shown for comparison. Numbers on graph show average percentage of reduction in absolute numbers compared with that in naive mice. Data from 1 or 2 experiments. $n=3-5$ mice per time point. 
A
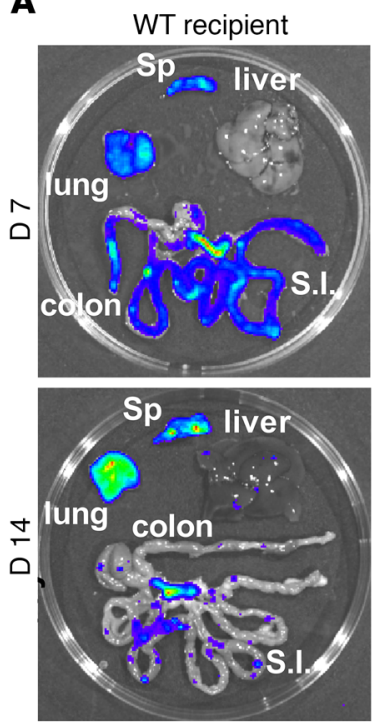

C

OWT

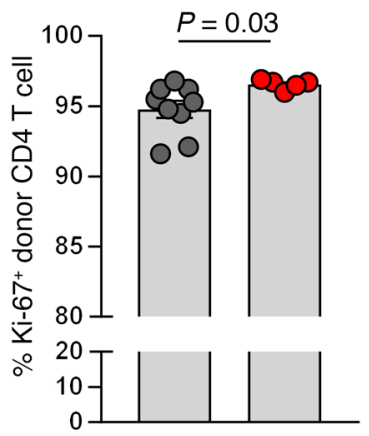

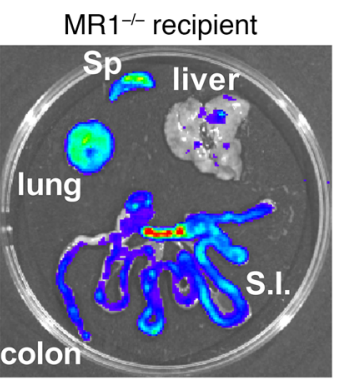

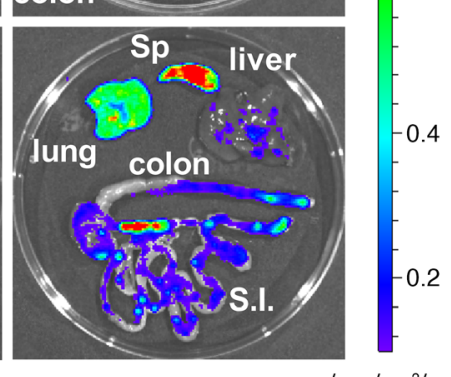

D

\section{OWT}

OMR1 ${ }^{-1-}$

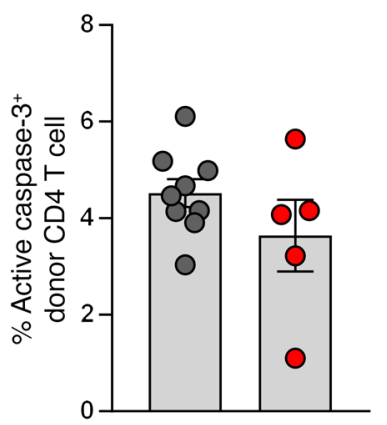

B

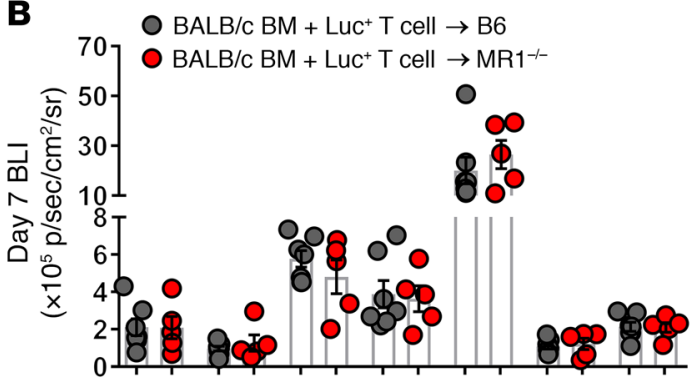

$1 \times 10^{5}$

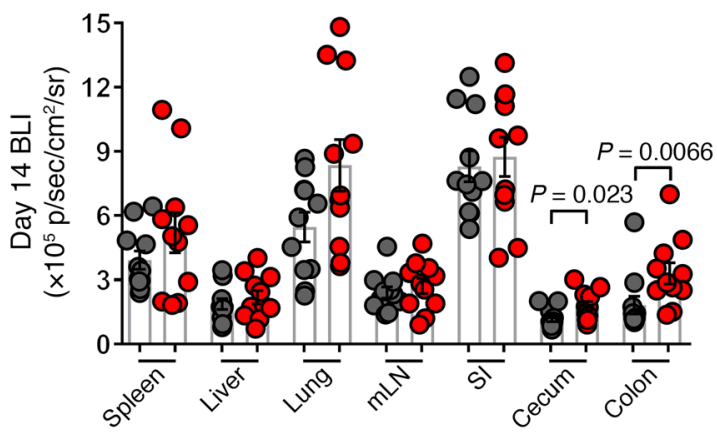

Figure 5. Recipient MAIT cells attenuate proinflammatory donor $\mathrm{CD4}^{+} \mathrm{T}$ cell expansion in the colon. (A) B6.WT and B6.MR1 $1 /$ - mice were lethally irradiated, transplanted with BALB/c.WT BM $\left(10 \times 10^{6}\right)$ and BALB/c.luciferase ${ }^{+}$T cells $\left(3 \times 10^{6}\right)$ and organs imaged at days 7 and 14 after transplant. Representative BLI images of the spleen, liver, lung, and $\mathrm{GI}$ tract (including $\mathrm{mLNs}$ ) are shown. (B) Quantitated BLI at days 7 (top) and 14 (bottom) is shown. Data are pooled from 2 independent experiments for each time point. Day 7, $n=5-7$ per group; day $14, n=11$ per group. (C) Frequency of proliferating donor $\mathrm{CD} 4^{+} \mathrm{T}$ cells in the colon lamina propria at day 14 , as determined by intracellular Ki-67 expression. (D) Frequency of apoptotic donor $\mathrm{CD} 4^{+} \mathrm{T}$ cells in the colon lamina propria at day 14 , as determined by intracellular active caspase-3 expression. Data combined from 2 replicate experiments are shown. $n=5-9$ per group. Data analyzed using the Mann-Whitney $U$ test.

ients. This also showed no difference in survival between the 2 groups (Figure 2, E and F), demonstrating that in these preclinical settings, it is recipient MAIT cells that abrogate GVHD.

Regulatory function of MAIT cells is confined to the GI tract. We established that recipient-derived MAIT cells appear to play a regulatory role during GVHD. To garner further understanding of the regulatory nature of MAIT cells in vivo, we analyzed serum cytokine levels in B6.WT and B6.MR1-deficient recipient mice after allogeneic SCT over a time course, with the aim of identifying when recipient MAIT cell activity might peak. Levels of serum IL-1 $\beta$, IL-4, IL-5, IL-6, TNF, IFN- $\gamma$, and GM-CSF were similar between $\mathrm{B} 6 . \mathrm{WT}$ and $\mathrm{B} 6 . \mathrm{MR}^{-/-}$recipients at day 4 after SCT (Figure 3A). A significant increase in IL-6 and TNF was observed at day 7 after SCT in B6.MR1 ${ }^{-/}$mice compared with B6.WT, but was not apparent at day 14 (Figure $3 \mathrm{~A}$ ), indicating the regulation provided by MAIT cells may be occurring in the early phase of GVHD. These data also suggested that the effect may be organ specific, as the serum cytokine levels in B6.MR1 ${ }^{-/-}$animals were unlikely to account for the significant reduction in survival observed.

We thus undertook semiquantitative histopathology of the liver, lung, SI, and colon of B6.WT and B6.MR1 ${ }^{-/-}$recipients after transplant. These analyses failed to identify any differences in the lung, liver, and SI (Figure 3, B-D). In contrast, pathology was sig- nificantly increased in the colon of B6. $\mathrm{MR}^{-/-}$recipients (Figure $3 \mathrm{E}$ ) and displayed perturbed barrier integrity (Figure $3 \mathrm{~F}$ ), suggesting GI tract-associated MAIT cells abrogated the pathology occurring in these tissues, thus improving survival. In order to regulate disease during GVHD, recipient MAIT cells must survive conditioning and the incoming alloreactive donor graft in the early transplant period. To establish whether this was indeed the case, we performed transplants in B6.WT mice and used the MR1 tetramer to identify recipient MAIT cells by flow cytometry. Recipient MAIT cells were identified in the SI and colon on day 3 and day 7 after transplant (Figure 4, A and B). Although their abundance was reduced compared with levels seen in naive mice, the relative reduction in recipient MAIT cells after BMT was equivalent to that of recipient $\gamma \delta$ T cells (Figure 4, A and B), another innate $\mathrm{T}$ cell population. Thus, recipient MAIT cells survive early after transplant with kinetics similar to those of other innate-like $\mathrm{T}$ cell populations.

Tregs play a key role in maintaining intestinal homeostasis (29), and their induction by particular microbiota $(30,31)$ and microbial-derived metabolites has been described $(32,33)$. We thus examined Tregs in the colon lamina propria in WT and $\mathrm{MR}^{-/-}$mice at day 14 after SCT. This revealed no difference in Treg frequency or number between the 2 groups (Supplemental 
A
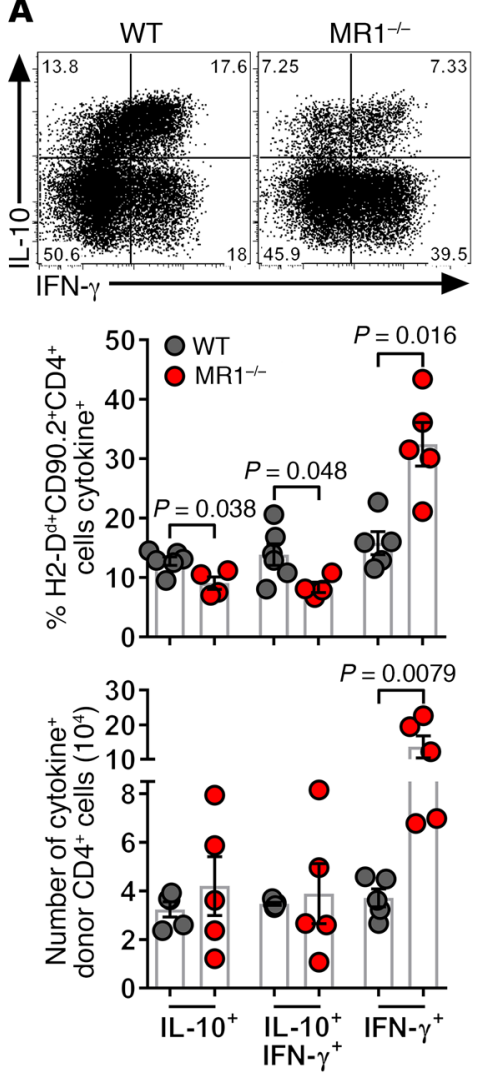

B
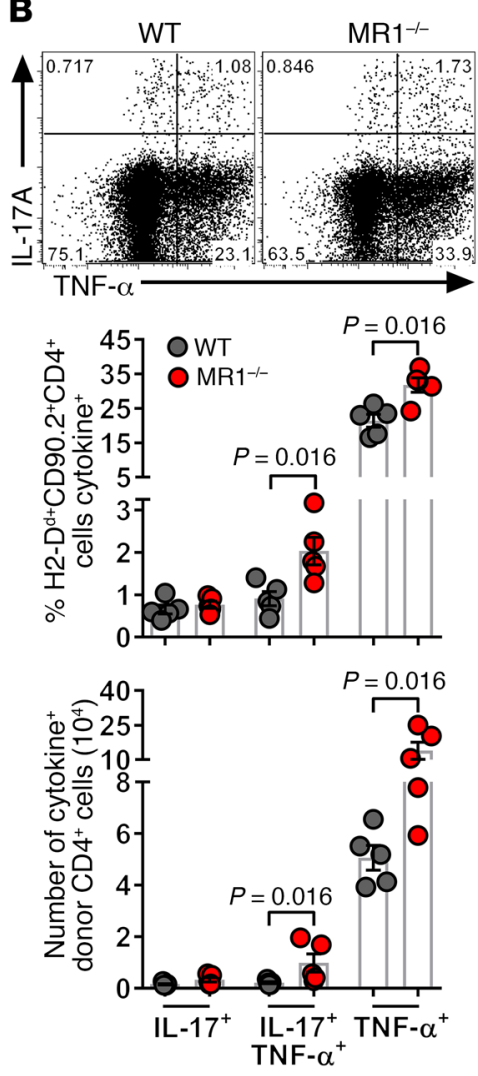

C
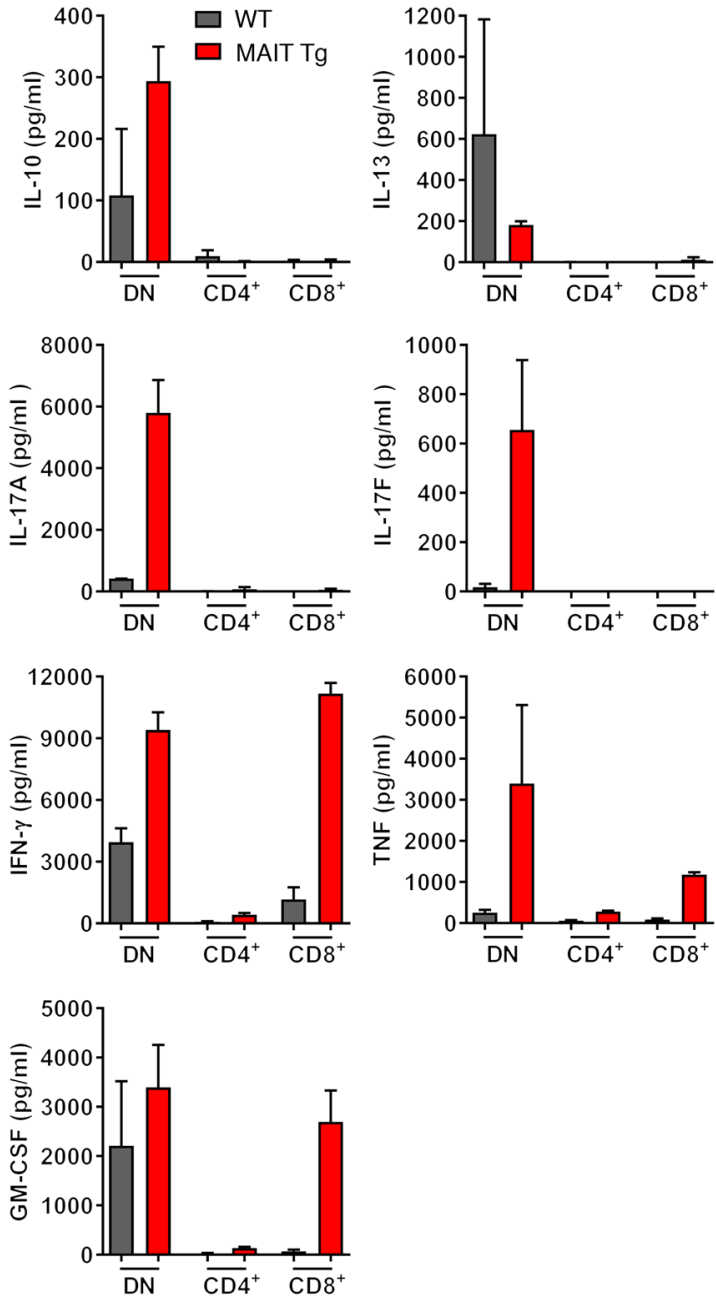

Figure 6. Recipient MAIT cells attenuate proinflammatory donor $\mathrm{CD4}^{+} \mathrm{T}$ cell expansion in the colon and secrete IL-17. B6.WT and B6.MR1 $1^{-/-}$mice were transplanted as in Figure 5A. Colon-derived lamina propria lymphocytes at day 14 after transplant were isolated and stimulated in vitro with PMA/ionomycin, followed by assessment of IFN- $\gamma$ and IL-10 production (A) and IL-17A and TNF- $\alpha$ production (B) by donor CD4+ cells using intracellular flow cytometry. Quantification of frequency (top) and absolute number (bottom) of cytokine-producing donor $\mathrm{CD} 4^{+} \mathrm{T}$ cells. Each point represents cells from pooled colons of 2 mice. Data from 2 independent experiments. $n=4-6$ mice per group. (C) $\mathrm{CD}^{+} \mathrm{CD}^{\text {neg }} \mathrm{CD}^{\text {neg }}(\mathrm{DN}), \mathrm{CD}^{+} \mathrm{CD}^{+} \mathrm{CD}^{\text {neg }}$, and $\mathrm{CD3^{+ }} C \mathrm{CD} 8^{+} \mathrm{CD} 4^{\text {neg }}$ cells were sort purified from B6.WT and B6.MAIT Tg LNs, stimulated in vitro with $\alpha-C D 3 / \alpha-C D 28$ for 72 hours, and cytokines determined in culture supernatant. Data from 2 independent experiments with 2-3 WT and 6-8 $\mathrm{MR}^{-/-}$mice pooled per group. Data analyzed using the Mann-Whitney $U$ test.

Figure 2), demonstrating that MAIT cells do not have a direct impact on Tregs and do not appear to regulate the severity of GVHD via this regulatory lineage.

To determine the effect recipient-derived MAIT cells have on donor $\mathrm{T}$ cell expansion after transplant, we conducted transplants using luciferase-expressing donor $\mathrm{T}$ cells injected together with (luciferase negative) BM into either B6.WT or B6.MR1-deficient recipients. This enabled us to quantify the level of donor $\mathrm{T}$ cell expansion in the presence or absence of MAIT cells in various organs at any given time point. Whole-body imaging at day 7 and day 14 after transplant did not show any difference in the bioluminescent imaging (BLI) signal of B6.WT and B6.MR1-- mice (data not shown). We thus conducted organ imaging at the same time points to look for organ-specific effects. The BLI signal originating from spleen, liver, lung, mesenteric LN ( $\mathrm{mLN}$ ), SI, cecum, and colon at day 7 was equivalent between B6.WT and B6.MR1 $1^{-1-}$ mice (Figure 5, A and B). In contrast, we observed a significant increase in the BLI signal of the cecum and colon in B6.MR1 ${ }^{-1-}$ mice compared with WT at day 14 after transplant (Figure 5, A and B). To determine whether the increased BLI in the colon was due to donor $\mathrm{T}$ cell migration and expansion and/or survival, we assessed donor $\mathrm{T}$ cell proliferation (Ki-67 expression) and apoptosis (active caspase-3 expression) in the colon lamina propria at day 14 after transplant. This revealed an increase in the frequency of proliferating $\mathrm{CD}^{+} \mathrm{T}$ cells in MR1-deficient mice compared with WT without a clear reduction in apoptosis (Figure 5, C and D). Differences in $\mathrm{CD} 8^{+} \mathrm{T}$ cell proliferation and apoptosis were not evident (Supplemental Figure 3, C and D). These data suggest that recipient MAIT cells constrain the alloreactive-driven expansion of donor $\mathrm{T}$ cells specifically in the colon. In support of this, we observed only minor differences in the frequencies or absolute numbers of $\mathrm{CD}^{+}$and no changes in $\mathrm{CD} 8^{+} \mathrm{T}$ cells in the spleen and mLNs of B6.WT and B6.MR1 ${ }^{--}$mice at day 7 or day 13 after transplant (Supplemental Figure 3, A and B). 
A
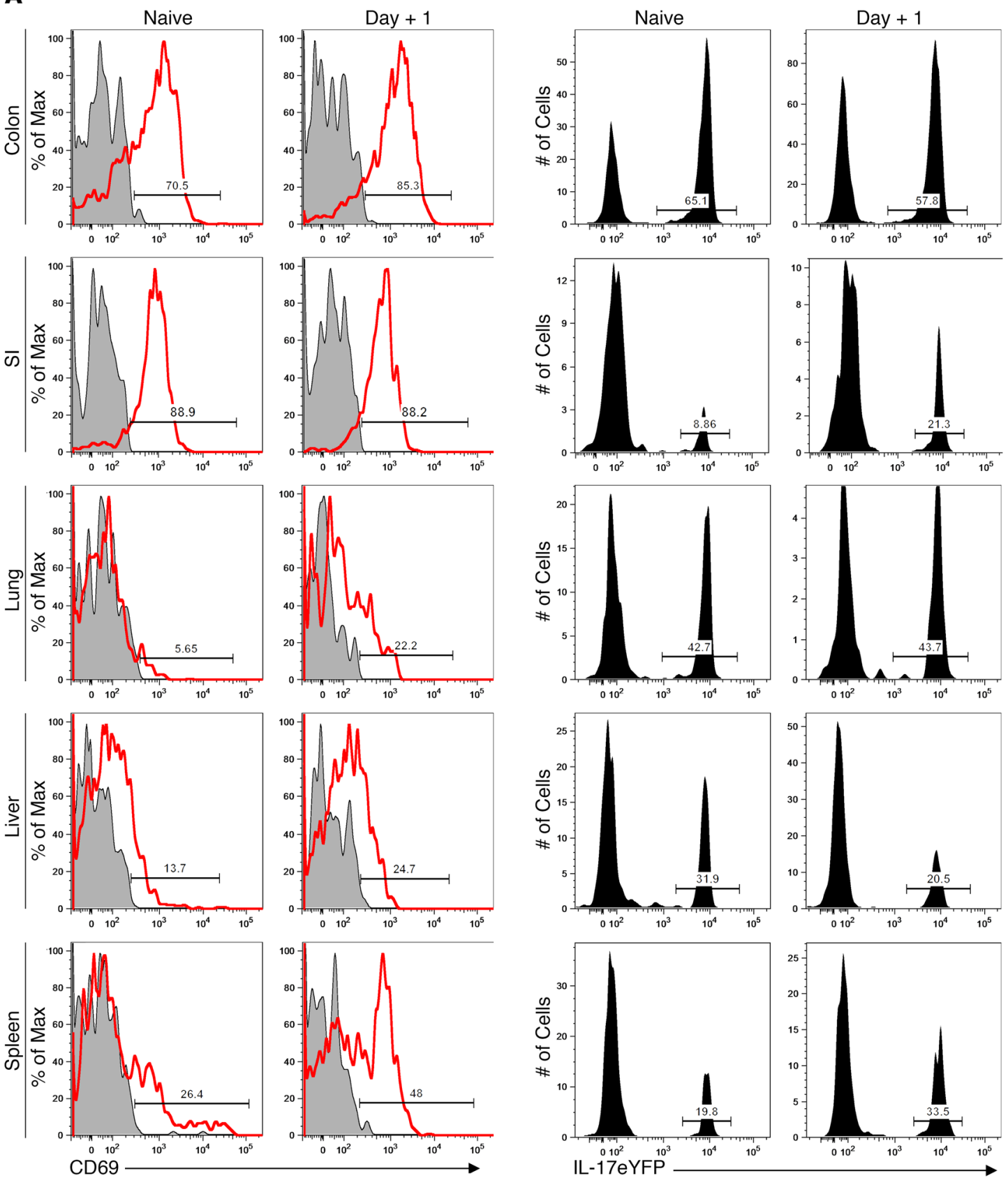

B
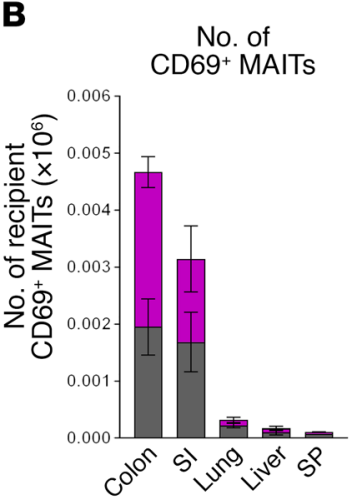

C
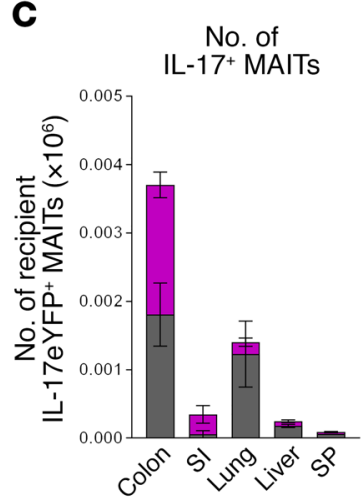

Figure 7. MAIT cells in the GI tract are constitutively activated in steady state and preferentially express IL-17 in the colon. (A) Representative histograms showing CD69 (red overlay; isotype, gray histogram) and IL-17eYFP (solid black) expression on recipient MAIT cells in the colon, SI, lung, liver, and spleen in steady-state and after allogeneic SCT $(\mathrm{d}+1)$. (B and C) Number of CD69+ and IL-17eYFP+ MAIT cells in tissues was quantified. Data pooled from 4 replicate experiments are shown. Colon, 3 experiments ( $n=6-12)$; SI, 3 experiments $(n=6-10)$; liver, 2 experiments ( $n=4-8)$; lung, 2 experiments ( $n=4-8)$; spleen, 2 experiments $(n=3-4)$. 


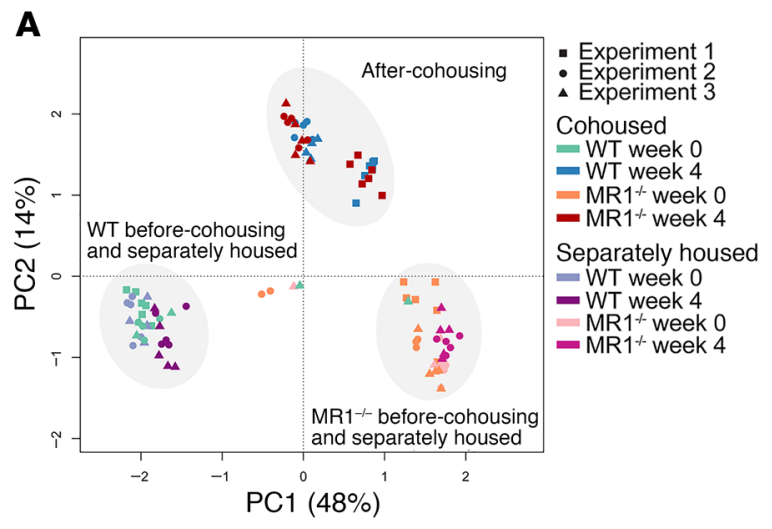

B

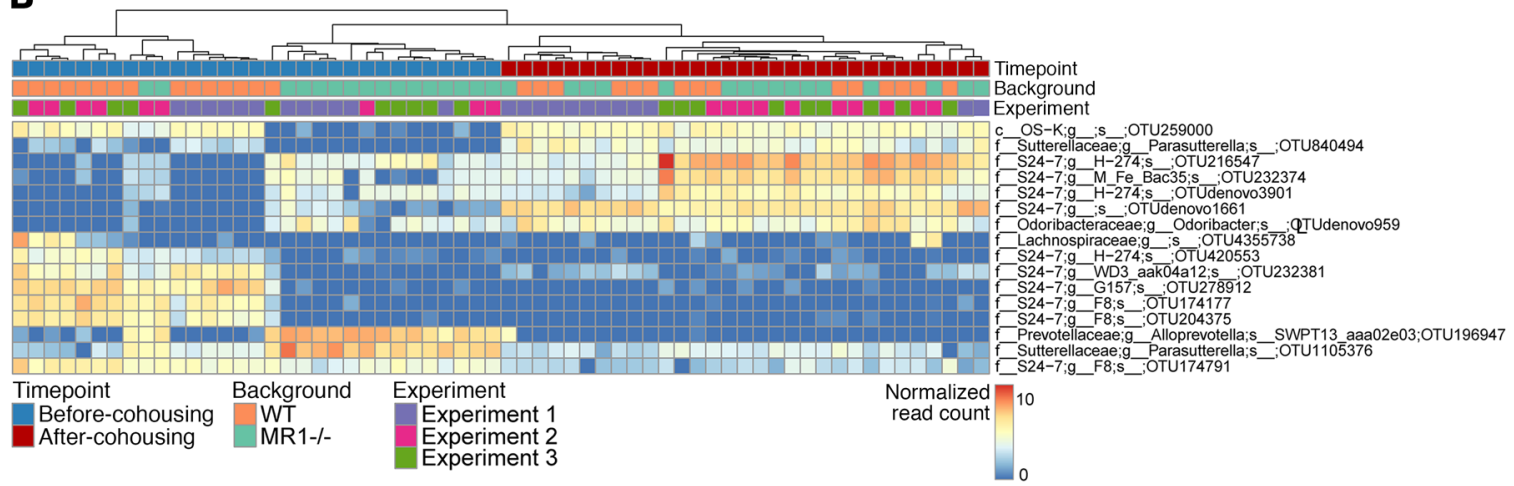

C

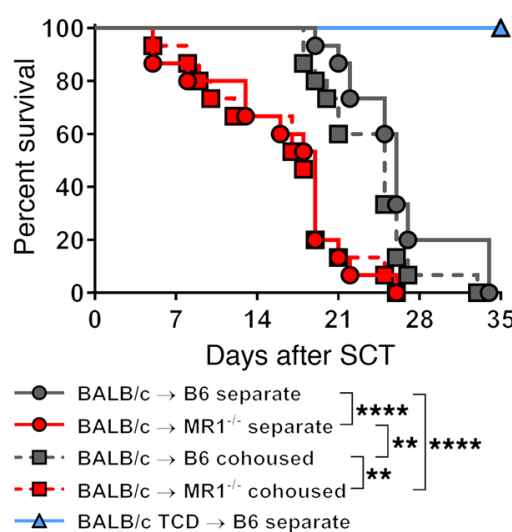

D

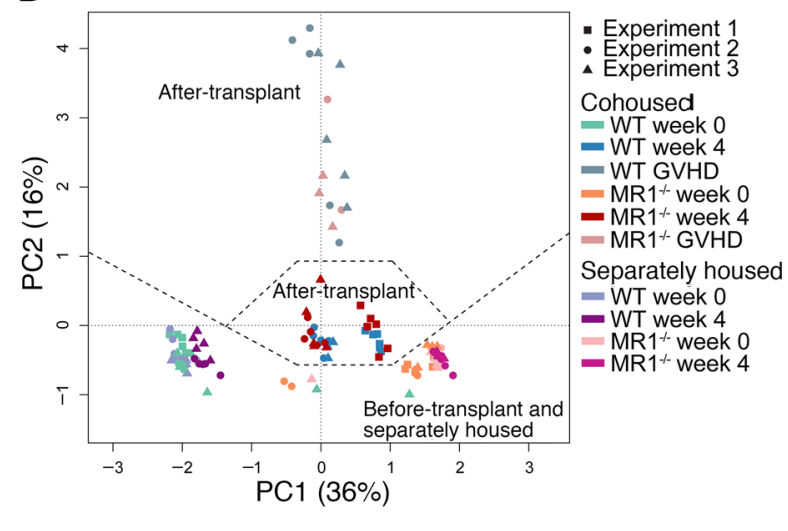

Figure 8. MAIT cells appear to shape the GI microbiome. (A) PCA plot of 165 rRNA-seq data generated from fecal samples collected from B6.WT and B6.MR1 $1^{-/-}$mice either before and after cohousing or when separately housed. Each dot represents an individual mouse, with symbols and colors corresponding to the genetic background and time point. Data combined from 3 independent experiments. Experiment $1, n=6$ mice per group; experiments 2 and 3, $n=5$ mice per group. (B) Heatmap showing significantly different OTUs between before and after cohousing samples in either B6.WT or B6.MR1 ${ }^{-1-}$ mice. OTUs displaying consistent movement in all 3 experiments are shown. Details contained in Supplemental Tables 1 and 2. (C) Separately housed or cohoused B6.WT and B6.MR1 ${ }^{-1-}$ mice were transplanted and clinical scores and survival monitored. Data pooled from 3 independent experiments. $n=15$ mice per group; TCD group, $n=9$. Survival data analyzed with a log-rank test. ${ }^{* * *} P<0.0001$; ${ }^{* *} P=0.0016$ (cohoused B6.WT versus cohoused B6.MR1 ${ }^{-I}$ ); ${ }^{*} P=0.0012$ (cohoused B6.WT versus separate B6.MR1 ${ }^{-1-}$ ). (D) PCA plot combining data from $\mathbf{A}$ with sequencing data from fecal samples collected after transplant. Data combined from 2 independent experiments. Experiments 2 and 3, $n=5-10$ mice per group. 16S rRNA-seq data analyzed using DESeq2.

To extend these findings, with the aim of establishing which proinflammatory population or populations may be suppressed by MAIT cells, we analyzed the cytokine production of donor T cells by intracellular cytokine staining. Small but significant increases in polyfunctional $\left(\mathrm{IFN}-\gamma^{+} \mathrm{IL}-17^{+}\right)$donor $\mathrm{CD} 4^{+} \mathrm{T}$ cells were seen in $\mathrm{mLNs}$ of B6.MR1 ${ }^{-/}$mice 7 days after transplant (Supplemental Figure 4, A and B). Subsequently, increased numbers of polyfunctional donor $\mathrm{CD}^{+} \mathrm{T}$ cells were observed in the lamina propria of colons from
B6.MR1 ${ }^{-/}$recipients (Figure 6, A and B), but not SI (Supplemental Figure 5, A-D). This included increased frequencies and numbers of IFN- $\gamma^{+}-$, IL-17+IFN- $\gamma^{+}-$, and TNF- $\alpha^{+}$-producing colonic donor $\mathrm{CD} 4^{+} \mathrm{T}$ cells. Concomitantly there was a small decrease in IL-10producing donor $\mathrm{CD}_{4}{ }^{+} \mathrm{T}$ cells in the colon of $\mathrm{MR}^{-/-}$mice (Figure 6A). This phenomenon was predominantly restricted to the CD4 lineage, as cytokine production by colonic $\mathrm{CD}^{+}$donor $\mathrm{T}$ cells was similar between B6.WT and B6.MR1 $1^{--}$mice (Supplemental Figure 
A

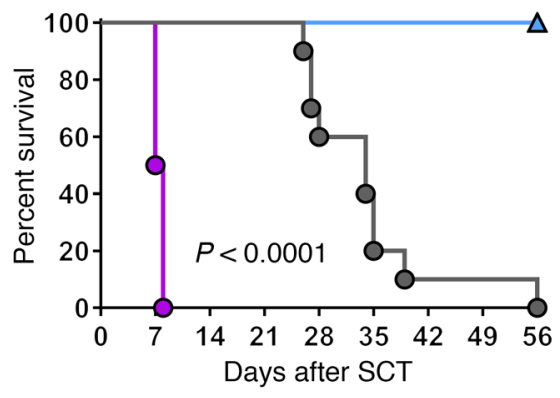

B

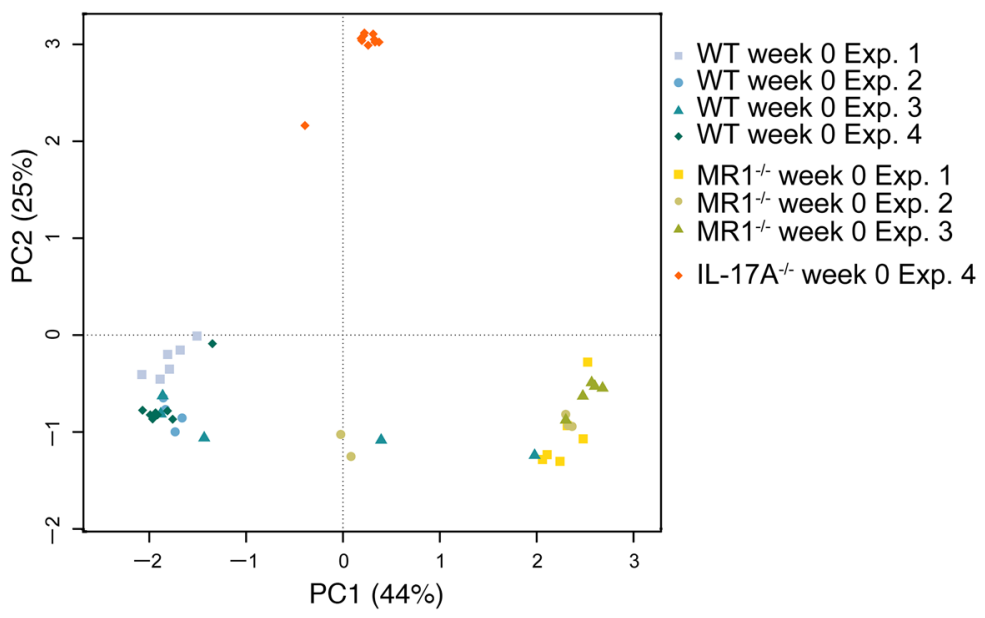

C

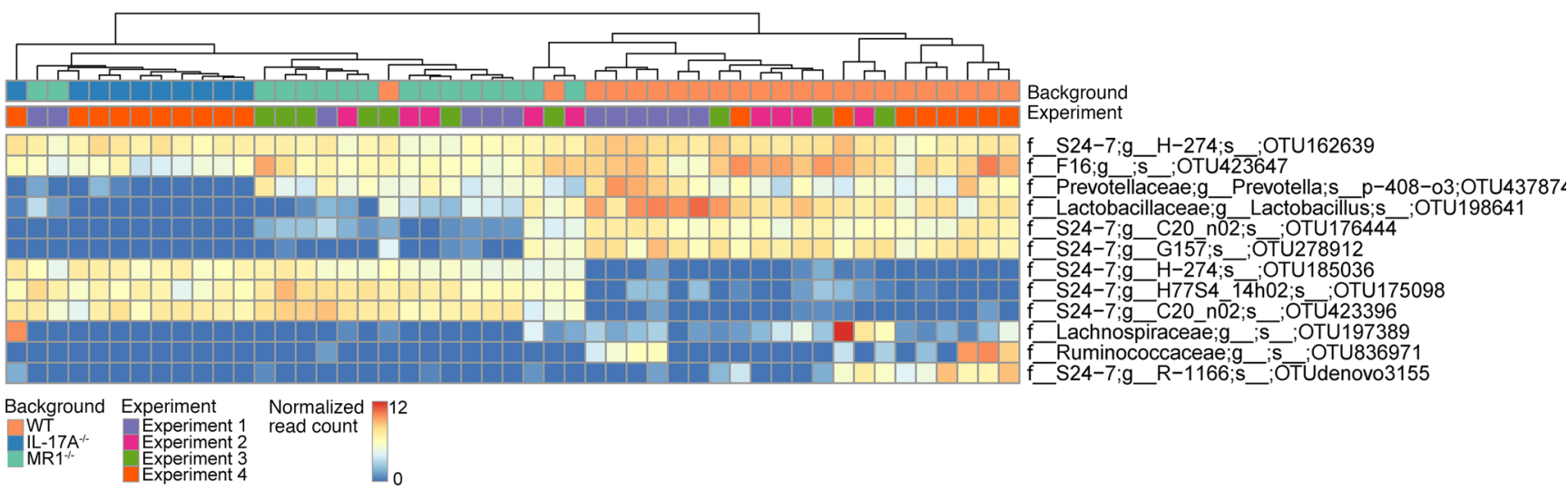

Figure 9. Analogous alterations in the microbiome in the absence of MAIT cells and IL-17A. (A) Separately housed B6.WT and B6.IL-17A ${ }^{-1-}$ mice were transplanted with $25 \times 10^{6} \mathrm{G}$-CSF-mobilized BALB/c splenocytes, and clinical scores and survival were monitored. Data pooled from 2 independent experiments ( $n=10$ per group; TCD group, $n=3$; B6.IL-17A ${ }^{-1-}$ TCD group, $n=5$ ). Survival data analyzed with a log-rank test. $P<0.0001$, B6.WT versus B6.IL-17A ${ }^{-1-}$. (B) Fecal samples collected from naive B6.WT and B6.IL-17A $\mathrm{A}^{-1-}$ mice were profiled by $16 \mathrm{~S}$ rRNA-seq. Principal component analysis of B6.WT, B6.IL-17A ${ }^{-1-}$, and B6.MR1-1- (Figure 7A data set) data demonstrates distinct OTU profiles. Each dot represents an individual mouse, with symbols and colors corresponding to the genetic background and experiment. Data from 4 experiments are shown. Experiment 1, WT versus MR1 $1^{-1-}, n=6$ mice per group; experiments 2 and 3 , WT versus MR1 ${ }^{-1-}, n=4-5$ mice per group; experiment 4, WT versus IL-17A ${ }^{-1-}, n=8-10$ mice per group. (C) Heatmap showing significantly different 0TUs between naive B6.IL-17A ${ }^{-/}$and B6.MR1 ${ }^{-/-}$mice in comparison with B6.WT mice from the same samples as used in B. Details contained in Supplemental Table 3. 165 rRNA sequencing data analyzed using DESeq2.

6, A and B). These data demonstrate that colon-associated MAIT cells suppress the expansion of polyfunctional, proinflammatory donor Th1 and Th17 cells in the colon during GVHD.

We next analyzed the cytokine profile of sorted $\mathrm{CD}^{+} \mathrm{CD} 4^{\text {neg- }}$ $\mathrm{CD} 8^{\text {neg }}, \mathrm{CD}^{+} \mathrm{CD}^{+}$, and $\mathrm{CD} 3^{+} \mathrm{CD} 8^{+} \mathrm{T}$ cells from WT and MAIT $\mathrm{Tg}$ mice, which contain only $\mathrm{V} \alpha 19^{+} \mathrm{V} \beta 6^{+} \mathrm{MR} 1$ tetramer-reactive $\mathrm{T}$ cells. MAIT cells produced significantly higher levels of IL-17A, IL-17F, IFN- $\gamma$, and TNF- $\alpha$, with the majority of cytokine production attributed to $\mathrm{CD}^{+} \mathrm{CD} 4^{\text {neg }} \mathrm{CD} 8^{\text {neg }}$ MAIT cells (Figure $6 \mathrm{C}$ ). These findings are consistent with recent reports using a similar MAIT $\operatorname{Tg}$ mouse $(9,14,19,25,34)$ or polyclonal murine $(7,21)$ or human MAIT cells (20) and highlight the significant IL-17 response generated by these cells.

Given that our data showed that the regulatory function of MAIT cells was restricted to the colon (Figure 3), we investigated why this may be the case by examining the activation status and IL-17 expression of MAIT cells from various target tissues in naive animals and early after SCT $($ day +1$)$ using IL-17eYFP fate reporter mice. Strikingly, MAIT cells in the GI tract displayed an activated phenotype with constitutive expression of CD69 at steady state (Figure 7, A and B). Analysis of IL-17 fate reporter expression (eYFP) further demonstrated that recipient MAIT cells in the colon expressed high levels of IL-17, even at steady state (Figure 7, A and C). This high number of activated MAIT cells expressing IL-17 in the colon is consistent with the preferential effects of this lineage specifically in the colon. Thus, MAIT cells in the colon, unlike those in any other organ examined, are both activated and high IL-17 producers at all times, representing a population poised to respond rapidly to bacterial metabolite antigens and regulate acute GVHD.

MAIT cells appear to regulate microbiome diversity in the GI tract. Previous studies have demonstrated that the microbiota and their microbial metabolites can profoundly influence intestinal inflammation during $\operatorname{GVHD}(35,36)$, and in a nontransplant setting, IL-17 has been shown to regulate this interplay (37). The data thus far 
have established that recipient MAIT cells reside in mucosal tissue and regulate GVHD in the colon. Given that the colon is a rich source of microbes and MAIT cells respond to microbial metabolites, we hypothesized that this cell population may respond to, and have an impact on, the gut microbiome. We thus cohoused B6.WT with $\mathrm{B} 6 . \mathrm{MR}^{-/-}$mice to equilibrate the intestinal microbiota prior to transplantation and confirmed that (a) MAIT cells do indeed influence the composition of the microbiota and that (b) this composition converges with WT after cohousing for a month (Figure 8, A and B, and Supplemental Tables 1 and 2). In contrast, separately housed mice of each strain analyzed in parallel retained their initial GI tract microbiota, confirming the community shift was a consequence of cohousing. We next transplanted cohoused mice and separately housed mice to establish whether the altered microbiota of the cohoused mice affected GVHD. B6.WT mice cohoused with $\mathrm{B} 6 . \mathrm{MR}^{-1-}$ mice showed survival kinetics equivalent to those of separately housed B6.WT mice (Figure 8C) and displayed a fecal microbial community similar to that of B6.MR1 ${ }^{-1}$ mice after transplant (Figure 8D), suggesting that any dysbiotic microbial populations present in $\mathrm{B} 6 . \mathrm{MR}^{-/-}$mice do not directly influence GVHD outcome.

Given that recipient MAIT cells produce very high levels of IL-17A and that IL-17A is known to be capable of regulating inflammatory colitis (38), we analyzed the importance of recipientderived IL-17 in GVHD. Indeed, B6.IL-17A ${ }^{-/-}$mice exhibited hyperacute GVHD, with a mean survival time of 7.5 days versus 34 days for B6.WT mice (Figure 9A). We confirmed that MAIT cells were indeed present in tissues from naive IL-17A $\mathrm{A}^{-/-}$mice (lung, SI, and colon) and in fact were in greater abundance in the colon compared with WT (Supplemental Figure 7, A and B), likely a response to the known dysbiosis in these animals (39).Furthermore, we showed that upon ex vivo expansion (Supplemental Figure 8) and activation, MAIT cells from both WT and IL-17A ${ }^{-/-}$mice expressed IFN- $\gamma$ and TNF, but not IL-10. As expected, only WT MAIT cells expressed IL-17A, demonstrating that MAIT cells from IL-17A ${ }^{-/-}$ mice were functionally active (Supplemental Figure 7, C and D). Comparison of the microbiota of $\mathrm{B} 6 . \mathrm{IL}^{-17 \mathrm{~A}^{-/}}$and $\mathrm{B} 6 . \mathrm{MR}^{-/-}$mice revealed that IL-17A depletion was associated with an additional disparate microbial composition (Figure 9B). Despite this, we were able to identify a small number of operational taxonomic units (OTU) displaying analogous abundance shifts in both deletion strains in comparison with B6.WT mice, thereby potentially representing populations controlled by similar, IL-17A-dependent mechanisms (Figure 9C and Supplemental Table 3). These data indicate that, in the absence of host-derived MAIT cells, a dysbiotic microbial community is established, some of which is IL-17A dependent, but this does not contribute directly to GVHD. The increased mortality of B6.MR1 $1^{-/-}$mice may instead be associated with the loss of an important recipient IL-17-producing cell, since this cytokine is clearly protective during the early phase of GVHD.

MAIT cells regulate barrier integrity and restrain alloantigen presentation and effector $T$ cell expansion. As increased mortality was observed in both MR1-deficient and IL-17A-deficient mice after SCT and we showed that MAIT-derived IL-17A expression differed between regions of the GI tract during steady state, we sought to determine the possible mechanisms of MAIT/IL-17mediated regulatory effects unique to the colon. We undertook RNA-sequencing (RNA-seq) analysis of colon and ileum tissue from naive $\mathrm{WT}, \mathrm{MR}^{-/-}$, and IL-17A ${ }^{-/-}$mice. Analysis of the differentially expressed genes in the colon revealed upregulation of 142 genes in MR1 $1^{-/-}$versus WT, with 67 common genes overlapping with upregulated genes seen in IL-17A ${ }^{-/-}$versus WT (Figure 10A) and 216 downregulated genes in $\mathrm{MR}^{-/-}$versus WT, with 103 common genes overlapping with downregulated genes observed in IL-17A ${ }^{-/-}$versus WT (Figure 10A). We next filtered out the differentially expressed genes common with ileum and examined the top 50 differentially expressed genes unique to colon. The heatmap shows clusters of differentially expressed genes common to both $\mathrm{MR}^{-/-}$and IL-17A ${ }^{-/-}$tissues compared with WT (Figure 10B), with selected genes of known function shown in Table 1. Strikingly, claudin $4(\mathrm{Cldn} 4)$ and claudin $8(\mathrm{Cldn} 8)$ gene expression was downregulated in both $\mathrm{MR}^{-/-}$and $\mathrm{IL}-17 \mathrm{~A}^{-/-}$mice compared with WT (Figure 10, B and C, and Table 1), suggesting MAIT cells may maintain barrier integrity in the colon by modulating tight-junction proteins via IL-17A, consistent with data shown in Figure 3F. Interestingly, gene expression of Semaphorin 6d and $4 \mathrm{~b}$ (Sema6d and $4 b$ ), important regulators of $\mathrm{T}$ cell immune responses (40, 41), was downregulated in $\mathrm{MR}^{-/-}$and $\mathrm{IL}^{-17 A^{-/-}}$ mice compared with WT (Figure 10B and Table 1), as was the expression of nuclear factor, interleukin 3 regulated (Nfil3) (Fig-

Table 1. Selected genes downregulated in both $\mathrm{MR}^{1 /-}$ and IL-17A ${ }^{-/-}$mice compared with WT

\begin{tabular}{|c|c|c|c|c|c|c|}
\hline \multirow[b]{2}{*}{ Gene } & \multirow[b]{2}{*}{ Symbol } & \multicolumn{2}{|c|}{ WT versus MR1 $1^{-1-}$} & \multicolumn{2}{|c|}{ WT versus IL-17A ${ }^{-/-}$} & \multirow[b]{2}{*}{ Function } \\
\hline & & (log) Fold change & $P$ value & (log) Fold change & $P$ value & \\
\hline \multicolumn{7}{|l|}{ Downregulated } \\
\hline Claudin 4 & Cldn4 & 1.01 & $2.01 \times 10^{-06}$ & 0.666 & 0.007 & Barrier integrity \\
\hline Semaphorin 6d & Sema6d & 0.432 & 0.011 & 0.438 & 0.005 & Regulator of $\mathrm{T}$ cell immune responses \\
\hline Semaphorin 4b & Sema $4 b$ & 0.568 & $5.48485 \times 10^{-06}$ & 0.49 & 0.0002 & Regulator of T cell immune responses \\
\hline Cathepsin L & Ctsl & 0.305 & 0.046 & 0.363 & 0.004 & Protease; Th17 differentiation \\
\hline UDP glucuronosyltransferase & Ugt1a8 & 4.705 & 0.008 & 13.08 & $1.3403 \times 10 \times 10^{-176}$ & Metabolism (glucuronidation pathway) \\
\hline
\end{tabular}

1 family, polypeptide A8

Data analyzed using edgeR with FDR $<0.05$. 
A

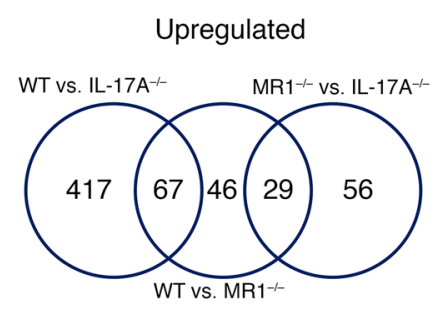

B

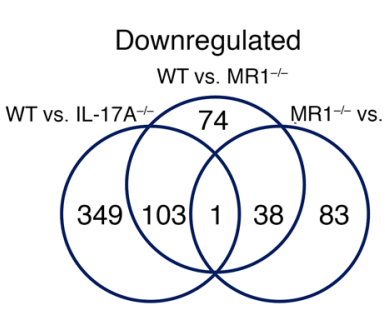

C

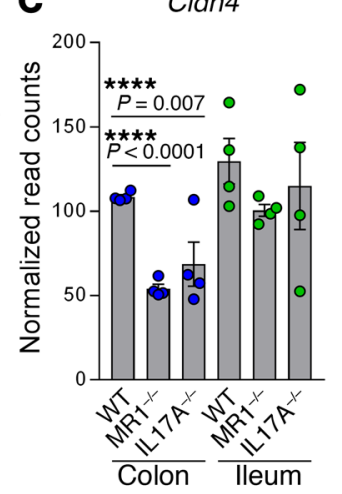

Cldn8

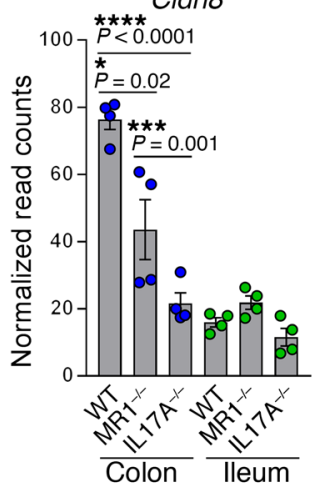

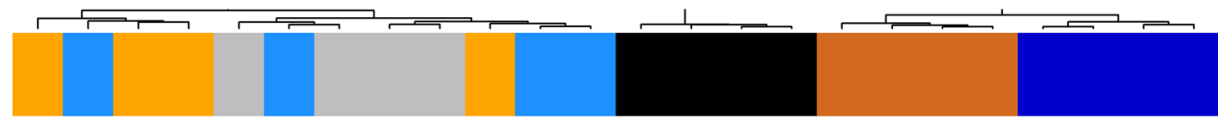

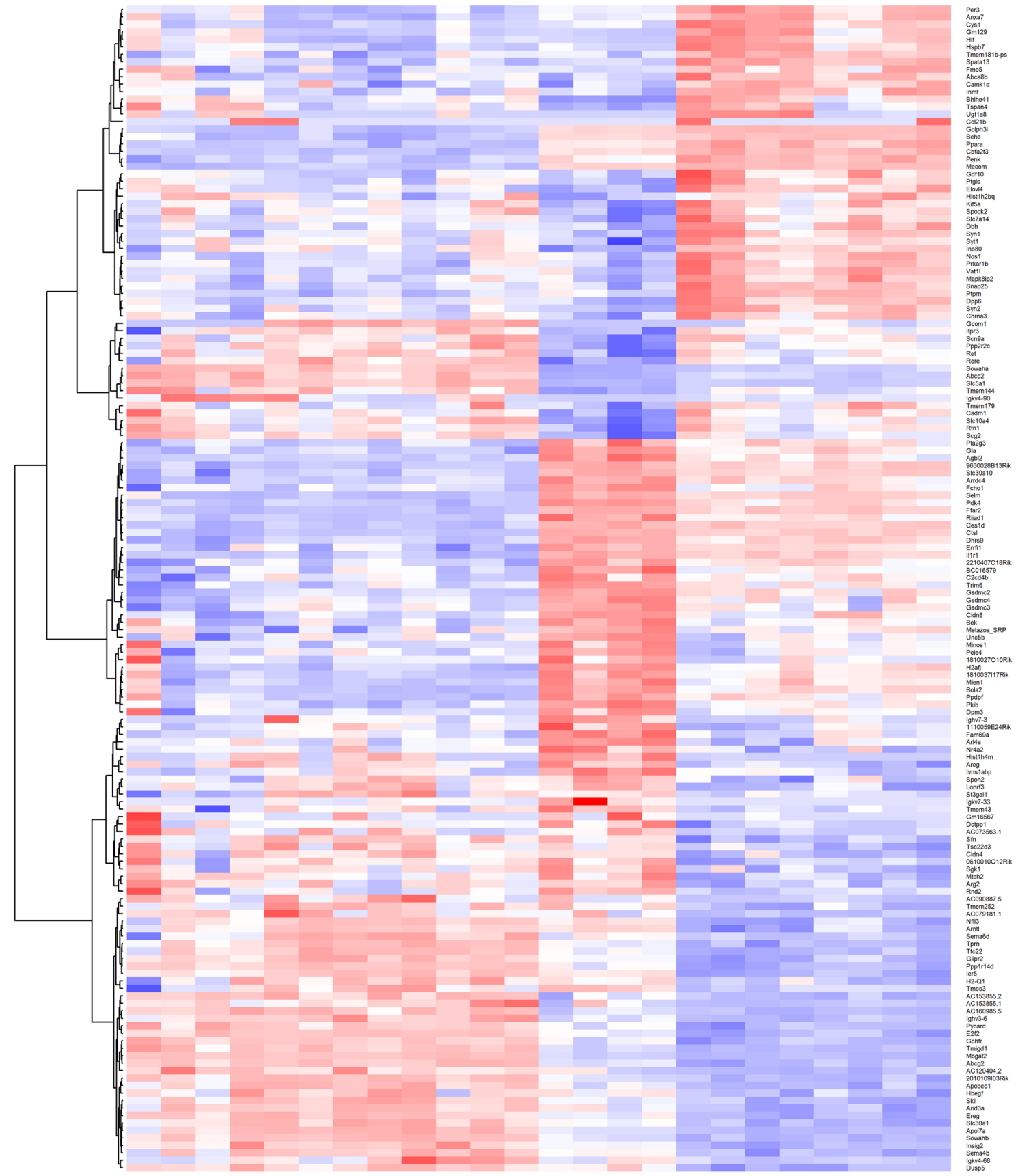


Figure 10. Regulation of host defense and metabolism genes by MAIT and IL-17A. RNA-seq analysis was performed on tissue from proximal colon and distal ileum of naive WT, MR1 $1^{-1-}$, and IL-17A ${ }^{-1-}$ mice ( $n=4$ per group). (A) Comparison of the number of overlapping genes differentially expressed within the colon; upregulated (upper) and downregulated (lower) genes. (B) Heatmap depicting differentially expressed genes comparing $\mathrm{MR}^{-1-}$ and IL-17A ${ }^{-/-}$versus WT colon, but not ileum. $\log _{2}$ transformed, normalized read counts were used. (C) Normalized read count data for claudin 4 and claudin 8 expression are shown. Data analyzed using edgeR with a false discovery rate $(\mathrm{FDR})<0.05$

ure 10B and Table 1), a transcription factor important in host immune defence against pathogens (42), and UDP glucuronosyltransferase 1 family, polypeptide A8 (Ugt1a8) (Figure 10B and Table 1), an enzyme important in the glucuronidation pathway of metabolism (43).

The accelerated mortality in $\mathrm{MR}^{-/}$- recipients in the third week after transplant with preferential GVHD in the colon suggested an enhancement in indirect antigen presentation by donor colonderived DCs within the $\mathrm{mLN}$, as we recently described (3). We thus undertook experiments to investigate the ability of recipient MAIT cells to regulate donor DC expansion and alloantigen presentation in the GI tract. In order to achieve this, we used TCR Tg Marilyn mice in which $\mathrm{CD} 4^{+} \mathrm{T}$ cells recognize the male $\mathrm{H}-\mathrm{Y}$ antigen in an $\mathrm{I}-\mathrm{A}^{\mathrm{b}}$-restricted fashion. We transplanted grafts comprising $\mathrm{BM}$ from B6.CD11c-GCDL mice (where the luciferase signal is used to track donor CD11 $\mathrm{c}^{+} \mathrm{DC}$ ) and B6.Marilyn TCR Tg T cells (to induce GVHD) into lethally irradiated male $\mathrm{B} 6 . \mathrm{WT}$ and $\mathrm{B} 6 . \mathrm{MR}^{-/-}$recipients (Figure 11A). Analysis of bacterial distribution by fluorescence in situ hybridization 14 days after SCT revealed increased bacterial translocation in the GI tract of $\mathrm{MRI}^{-/-}$recipients (Figure $11 \mathrm{~B})$, an effect also seen in $\mathrm{IL}-17 \mathrm{R}^{-/-}$recipients (39). Consistent with increased DAMP signaling in these animals, a significant expansion of donor DC (bioluminescence intensity) in the $\mathrm{mLN}$ in MR1-deficient mice was also observed (Figure 11C), confirming that recipient MAIT cells can indeed regulate GI tract integrity and donor DC expansion and/or migration in the GI tract.

To ascertain whether this donor DC expansion in MR1deficient mice would lead to an increase in $\mathrm{T}$ cell priming and effector $\mathrm{T}$ cell expansion, we transplanted B6.WT BM with B6.Marilyn TCR Tg T cells into lethally irradiated male B6.WT and $\mathrm{B} 6 . \mathrm{MR}^{-/}{ }^{--}$recipients. On day 12 after SCT, we transferred Marilyn $\mathrm{Tg}_{\mathrm{luc}} \mathrm{C}^{+} \mathrm{T}$ cells (here the luciferase signal reports for donor alloantigen presentation and subsequent antigen-specific $\mathrm{T}$ cell expansion; Figure 11D). Alloantigen-specific (Marilyn Tg luc ${ }^{+}$) $\mathrm{T}$ cells were preferentially expanded in the GI tract of MR1-deficient mice (Figure 11, E and F), with an increased frequency of pathogenic IFN- $\gamma^{+} \mathrm{IL}-17^{+}$-secreting effector T cells (Figure 11, G and H). Collectively, these data demonstrate that recipient MAIT cells have the ability to suppress alloantigen presentation by donor DC and subsequent effector $\mathrm{T}$ cell expansion following transplantation, culminating in the attenuation of GVHD.

\section{Discussion}

The importance of mucosal immunity and responses to microbiota at environmental interfaces in disease is only now beginning to be appreciated. MAIT cells, whose primary role at steady state appears to be antimicrobial, are likely to be important in modulat- ing inflammatory diseases that target mucosal surfaces $(16,44)$. Previous work has demonstrated both inflammatory and regulatory functions for MAIT cells in various mouse models and human disease $(8,9)$; however, the function of MAIT cells in the context of GVHD has not been studied. Using MAIT-deficient mice and MR1 tetramers to specifically identify endogenous MAIT cells in nongenetically modified mice, we have shown that recipientderived MAIT cells persist in the colon following total body irradiation. Here, lamina propria MAIT cells appear to control GVHD by regulating barrier function and attenuate proinflammatory cytokine production by donor $\mathrm{CD}^{+} \mathrm{T}$ cells. This is associated with shifts in the intestinal microbiota.

MAIT cell activation requires presentation of riboflavin-based precursors captured by the MHC class I-related molecule MR1 (11-15). The breakdown of epithelial barrier integrity after BMT, a result of chemoradiotherapy during conditioning and GVHD itself (4), is likely to increase access of T cells and APCs to bacteria (2) that utilize the riboflavin biosynthesis pathway. Subsequent capture, processing, and presentation of metabolite antigen on MR1 to recipient MAIT cells will lead to cellular activation and effector function. In addition, MAIT cells can also be activated in an antigen-independent manner by IL-12 and IL-18 (45-47). The end result of MAIT cell activation is to limit microbial colonization and disease by potential pathogens. In the context of GVHD, it appears that while multiple immune pathways are in effect, MAIT cells play a notable and nonredundant role. The present study demonstrates a link between MAIT cells and the composition of the gut microbiome in the mouse at steady state. In particular, shifts in the abundance of several OTUs from the bacterial family S24-7, recently defined as "Candidatus Homeothermaceae" (48), are notable in mice lacking MAIT cells. However, while the microbiome profile of $\mathrm{B} 6 . \mathrm{MR}^{--}$mice can be transferred to B6.WT mice through cohousing, these communities do not appear to influence GVHD. This suggests that the transferrable microbiota, putatively regulated by MAIT cells, likely those with an active riboflavin biosynthesis pathway, are not directly pathogenic in the context of GVHD if MAIT cells are present.

The mucosal location of MAIT cells enables rapid sensing of any breakdown in mucosal surface integrity and subsequent bacterial and/or PAMP molecule translocation, a prominent feature of GVHD. The mechanism by which MAIT cells are able to suppress inflammation may be directly through cytokines, as we and others have demonstrated their ability to generate large amounts of cytokines, particularly IL-17A $(6,7,14)$. The majority of adult MAIT cells exhibit a mature and differentiated phenotype (7), suggesting they are primed for rapid cytokine production following stimulation. Notable expression of IL-17 is related to the expression of high levels of ROR $\gamma \mathrm{t}$ and low levels of T-bet (6, 7). MAIT cell-derived cytokines, particularly IL-17, induce recruitment of myeloid cells, such as monocytes/macrophages and neutrophils that mediate pathogen clearance, as demonstrated in other disease models (49-51).

The GI tract contains numerous sources of IL-17 of both hematopoietic and nonhematopoietic origin. Nonhematopoietic sources of IL-17 in the GI tract include Paneth cells, which have been shown to play a role in inflammation (52), while T cell sources include $\alpha \beta, \gamma \delta$, NKT, innate lymphoid, and MAIT cells (5). IL-17 
has direct effects on maintaining epithelial barrier integrity. In conjunction with IL-22, IL-17A and IL-17F enhance production of antimicrobial peptides (53). Furthermore, following colitis damage induced by dextran sulfate sodium (DSS), epithelial cells exposed to IL-17 and the growth factor FGF2 undergo proliferation to restore barrier function and prevent inflammatory bacterial accumulation (54). IL-17 derived from $\gamma \delta$ T cells has also been shown to increase expression of occludin, a tight junction protein critical for maintaining homeostasis of epithelial cell permeability (55). As such, lack of IL-17 leads to increased susceptibility to infection by both bacteria and fungi (56-60). Therefore, IL-17 is required to maintain epithelial cell barrier function, and perturbation of IL-17 in this context can lead to enhanced inflammation and GI damage. It is thus likely that the protection from GVHD in the GI tract by MAIT cells reflects their role in maintaining mucosal integrity and that the enhanced donor $\mathrm{T}$ cell responses seen in the colon in their absence reflect enhanced PAMP signaling locally within the GI tract. This pathway has recently been shown to be critical in determining the severity of GVHD after BMT $(2,3)$. Given that MAIT cells are but one source of IL-17A in the GI tract, it is thus not surprising that their absence results in a phenotype intermediate between $\mathrm{IL}-17 \mathrm{~A}^{-/-}$and WT recipients. Thus, the exacerbation of GVHD in the absence of IL-17A clearly involves multiple additional pathways over and above those mediated by MAIT cells. The pathways of regulation utilized by MAIT cells and IL-17A can only be definitively dissected by the use of cre-based systems that are not yet available, but will enable the specific removal of cytokines from MAIT cells. That said, given the known ability of IL-17A from conventional donor $\mathrm{T}$ cells to promote acute GVHD, including within the GI tract $(39,61,62)$, we do not envisage that the administration of this cytokine to patients would be safe.

It is important to note that MAIT cells are a rare $(<1 \%)$ population in relatively clean inbred mice (7) in contrast with humans, where they represent up to $5 \%$ of peripheral blood T cells and $45 \%$ of hepatic T cells (20). Thus, the clear propensity to acute GVHD in the GI tract in mice lacking MAIT cells is likely to underestimate their importance in patients undergoing BMT. In fact, recipient MAIT cells have recently been shown to be resistant to myeloablative chemotherapy prior to autologous SCT. Importantly, pretransplant MAIT cell numbers were predictive for reductions in subsequent infection and inflammatory responses (63), consistent with the findings here after allogeneic SCT. The importance of GI tract integrity and microbiome-derived metabolites in modulating GVHD have been demonstrated as a very early event after BMT $(36,64)$. Since the numbers of donor MAIT cells in the bone marrow and splenic $\mathrm{T}$ cell-derived grafts in these experiments are very limited, MAIT cell reconstitution primarily reflects bone marrow-derived MAIT cells, which are selected in the thymus of MR1-bearing double-negative thymocytes (65). This reconstitution is thus slow (beyond 3 to 4 weeks) and develops after GVHD in the GI tract has already occurred. For these reasons, the effect of donor MAIT cells is minimal in these preclinical systems, but we acknowledge this may not necessarily be true in clinical transplantation, where the number of MAIT cells in blood is much higher. Indeed, a recent study has now demonstrated an association between donor MAIT cell numbers and gut microbiota after transplantation (66). Now that reagents are available to study what is a very large subset of $\mathrm{CD}^{+} \mathrm{T}$ cells in humans, it will be important to ascertain whether numerical and/or functional defects in this population correlate with alterations in the intestinal microbiome and GVHD outcomes in patients.

\section{Methods}

Mice. Female C57BL/6J (B6.WT, H-2 $)$, B6D2F1 (H-2 $\left.{ }^{\mathrm{b} / \mathrm{d}}\right)$, and BALB/c $\left(\mathrm{H}-2^{\mathrm{d}}\right)$ mice were purchased from the Animal Resources Centre (Perth, Australia). $\mathrm{MR}^{-/-}$mice on a C57BL/6 background (67) and iV $1919 / \mathrm{V} \beta 6$ double-Tg mice on a $\mathrm{C}^{-1-} \mathrm{C} 57 \mathrm{BL} / 6$ background (68) (B6.MAIT Tg) were provided by O. Lantz. B6.Marilyn TCR Tg mice (on a Rag2 ${ }^{-/}$background) (69) were provided by P. Matzinger (NIH, Bethesda, Maryland, USA). To generate Marilyn TCR Tg luciferase ${ }^{+}$mice, B6.Marilyn TCR $\mathrm{Tg}$ mice were crossed with the B6. $\beta$-actin-luciferase (CD90.1+) line (70) (provided by R Negrin, Stanford University, Stanford, California, USA) to generate Marilyn TCR Tg and luciferase ${ }^{+}$CD90.1 homozygous mice on a B6.Rag2 ${ }^{-/}$background. B6.CD11c-GCDL mice (71) (provided by G. Hammerling, German Cancer Research Centre, Heidelberg, Germany), B6.IL-17Cre $\times$ B6.ROSA26eYFP mice (IL-17eYFP) (72) (provided by B. Stockinger, Francis Crick Institute, London, United Kingdom; and F. Costantini, Columbia University, New York, New York, USA), BALB/c luciferase ${ }^{+}$mice (73) (provided by R. Negrin), LP/J mice (Jackson Laboratories, catalog 000676), and BALB/c CD45.1 ${ }^{+}$mice (sourced from the Peter MacCallum Cancer Centre, Melbourne, Australia) were bred in house. We used age-matched mice in all experiments. Mice were housed in microisolator cages and received acidified autoclaved water (pH 2.5) after BMT.

Stem cell and BMT. Total body irradiation (1000 cGy, B6 background; 1100 cGy, B6D2F1) was administered on day -1 (137Cs source at 82 cGy/ $\mathrm{min})$, split into 2 doses separated by 3 hours. Lethally irradiated mice were injected intravenously on day 0 with either 10 or $25 \times 10^{6}$ whole or TCD splenocytes from donor mice pretreated for 6 days with G-CSF (10 $\mu \mathrm{g} / \mathrm{d})$. $\mathrm{T}$ cell depletion was performed by incubating splenocytes with hybridoma supernatants containing anti-CD4 (RL172), anti-CD8 (TIB211), and Thy1.2 (HO-13-4), followed by incubation with rabbit complement (Cedarlane). Alternatively, lethally irradiated mice were injected intravenously on day 0 with 5 to $10 \times 10^{6} \mathrm{BM}$ cells and 2 to $5 \times 10^{6}$ enriched splenic T cells $\left(80 \%-90 \% \mathrm{CD}^{+}\right)$. TCD grafts containing only $10 \times 10^{6}$ TCD BM were transplanted as non-GVHD controls.

Assessment of GVHD. The degree of systemic GVHD was assessed using a cumulative scoring system that measures changes in 5 clinical parameters: weight loss, posture (hunching), activity, fur texture, and skin integrity (maximum index, 10). Mice were monitored daily, and those with GVHD clinical scores of 6 or more (74) were sacrificed and the date of death deemed as the next day in accordance with institutional animal ethics guidelines. Organ pathology was determined by blinded assessment of formalin-fixed, paraffin-embedded, H\&Estained sections as described previously (39).

Flow cytometry. CD4 (clone RM4-5), V $\beta 6$ (clone RR4-7), Ki-67 (clone B.56), active caspase-3 (clone C92-605), and CD25 (clone 7D4) were all purchased from BD Biosciences. CD90.1 (Thy1.1, clone HIS51) was purchased from eBiosciences. CD3 (clone 1452C11), CD8 (clone 53-6.7), CD45.1 (clone A20), CD45.2 (clone 104), TCR $\gamma \delta$ (clone GL3), CD326 (clone G8.8), CD19 (clone 6D5), CD90.2 (Thy1.2, clone 53-2.1), CD69 (clone H1.2F3), H2D (clone 34-2-12), FOXP3 (clone 150D), IL-10 (clone JES5-16E3), IL-17A (clone TC118H10.1), IFN- $\gamma$ (clone XM1.2), and TNF (clone MP6-XT22) were all 
A

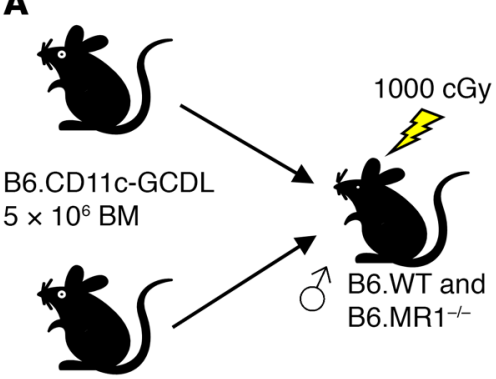

B6.Marilyn TCR Tg (CD90.2+)

$0.1 \times 10^{6} \mathrm{CD} 4 \mathrm{~T}$
B

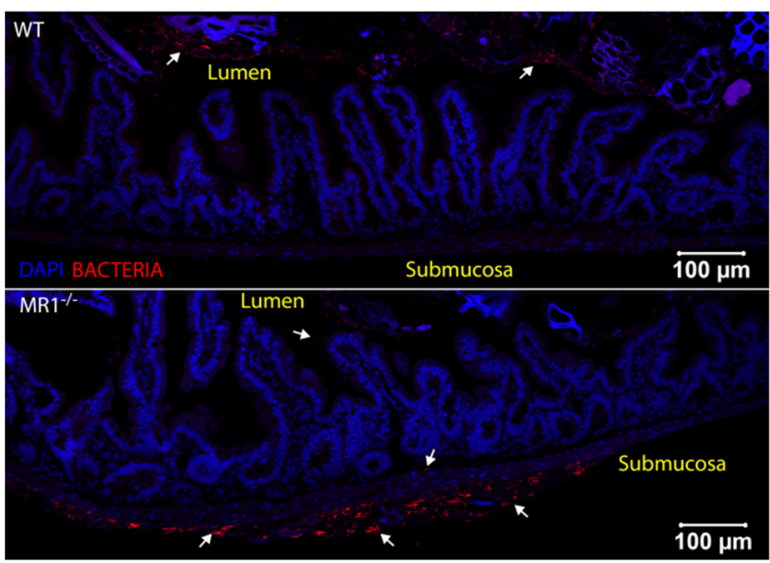

C

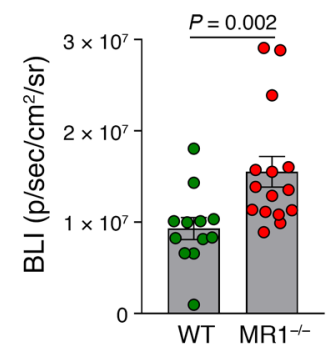

D

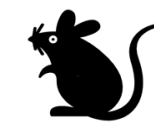

B6.WT

$5 \times 10^{6} \mathrm{BM}$
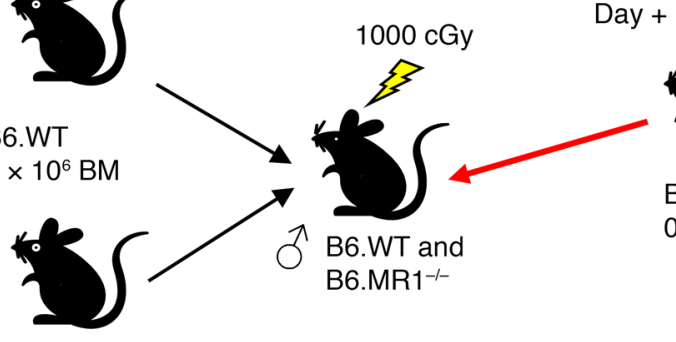

B6.Marilyn TCR Tg $\times$ B6.luciferase ${ }^{+}\left(\right.$CD90.1 $\left.1^{+}\right)$

$0.2 \times 10^{6} \mathrm{CD} 4 \mathrm{luc}^{+} \mathrm{T}$

B6.Marilyn TCR Tg (CD90.2+)

$0.05-0.2 \times 10^{6} \mathrm{CD} 4 \mathrm{~T}$

E

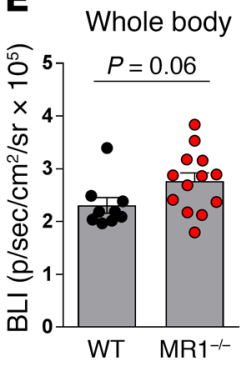

G

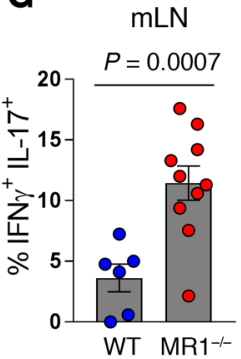

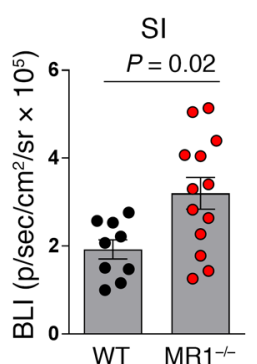

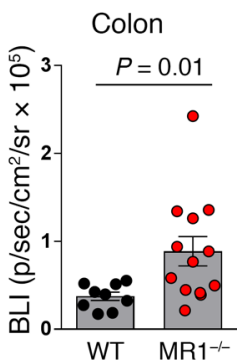

H

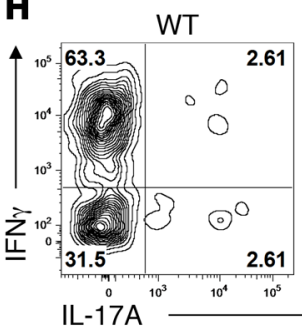

$\mathbf{F}$

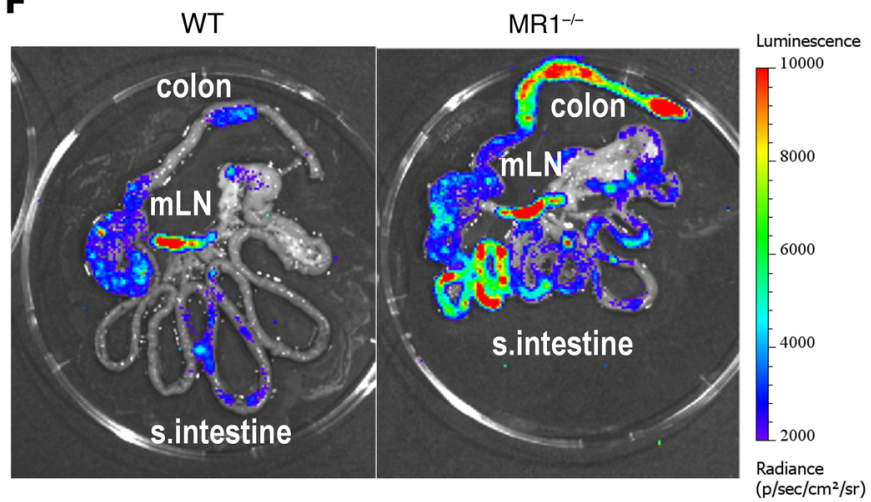

Figure 11. Recipient MAIT cells suppress alloantigen-specific Marilyn TCR Tg effector T cell expansion. (A-C) Lethally irradiated male B6.WT and B6.MR1 ${ }^{-1-}$ recipients were transplanted with TCD B6.CD11C-CCDL BM and B6.Marilyn TCR Tg T cells. Bacterial translocation in ileum tissue by FISH at day 14 after allogeneic SCT is shown in B. Individual organs were imaged on day 14 after allogeneic SCT, and BLI data combined from 3 replicate experiments are shown in C ( $n=12-15$ per group). (D-H) Lethally irradiated male B6.WT and B6.MR1 $1^{-1-}$ recipients were transplanted with B6.WT BM and B6.Marilyn TCR Tg T cells, followed by transfer of Marilyn luc ${ }^{+} \mathrm{T}$ cell on day 12 after allogeneic SCT. Individual organs were imaged 7 days after transfer, and BLI data combined from 3 replicate experiments are shown in $\mathbf{E}\left(n=9-13\right.$ per group). Representative images are shown in $\mathbf{F}$. Frequency of pathogenic IFN- $\gamma^{+} I L-17^{+}$Marilyn luc $c^{+}$cells in $\mathrm{mLN}$ was determined, and data combined from 2 replicate experiments are shown in $\mathbf{G}(n=5-10$ per group). Representative FACS plots are shown in $\mathbf{H}$. Data analyzed using the Mann-Whitney $U$ test.

purchased from BioLegend. Fixation and permeabilization were undertaken for intracellular staining (BD Fix/Perm Kit; BD Biosciences) and nuclear staining (Fix/Perm Kit; eBiosciences) according to the manufacturer's instructions. The LIVE/DEAD Fixable Aqua
Dead Cell Staining Kit (Invitrogen) was utilized to gate viable cells. Cells were acquired on a BD LSRFortessa and analyzed with FlowJo V9 (Treestar). MR1 tetramers were prepared and used as previously described $(7,8,11,75)$. 
Cytokine bead array. Serum and culture supernatant cytokine levels were measured using mouse Flex Array sets (BD Biosciences) according to the manufacturer's instructions.

Barrier integrity assay. Gut permeability was determined by FITC-dextran assay, as described previously (39). Bacterial translocation in tissues by FISH was performed as described previously (39).

Xenogen imaging. Donor T cell and DC expansion in vivo was measured by luciferase signal intensity using the IVIS Spectrum Imaging System (Caliper Life Sciences). Mice were injected with luciferin (0.5 $\mathrm{mg}$ ) subcutaneously and imaged after 5 minutes. Individual organs were harvested and imaged after reinjection with luciferin. Data were analyzed using Living Image software (PerkinElmer). Light emission is presented as photons per second per $\mathrm{cm}^{2}$ per steer radiant $\left(\mathrm{ph} / \mathrm{cm}^{2} / \mathrm{s} / \mathrm{sr}\right)$.

Lymphocyte isolation from tissues. Lymphocytes were isolated from colon and SI using the gentleMACS Dissociator and Mouse Lamina Propria Dissociation Kit (both Miltenyi Biotech) according to the manufacturer's instructions. Lymphocytes were isolated from lung tissue by digestion with collagenase III as described previously (76) and from liver tissue by mechanical disruption and Percoll density centrifugation as described previously (77).

MAIT cell expansion ex vivo. This was performed as described previously (7). Briefly, spleens and thymus from naive B6.WT and IL-17A ${ }^{-/-}$mice were harvested, mashed, and erythrocytes lysed using Gey's solution. Cells were resuspended in IMDM supplemented with $152 \mathrm{nM}$ MR1 ligand (5-OP-RU synthesized in DMSO; ref. 78), $50 \mathrm{ng} /$ $\mathrm{ml}$ recombinant human IL-2 (Proleukin; Prometheus Laboratories, Bayer HealthCare Pharmaceuticals), 10\% FBS, L-glutamine (2 mM), penicillin (100 units/ml), streptomycin $(100 \mu \mathrm{g} / \mathrm{ml})$, sodium pyruvate $(1 \mathrm{mM})$, NEAA $(1 \times)$, and $2-\beta$-mercaptoethanol $(23 \mu \mathrm{M})$. After 5 days culture, cells were collected and stimulated with phorbol myristate acetate $(50 \mathrm{ng} / \mathrm{ml})$ and ionomycin $(500 \mathrm{ng} / \mathrm{ml})$ in fresh culture medium for 18 hours (brefeldin A added in the final 4 hours). Activated cells were then collected and stained for intracellular IFN- $\gamma$, TNF, IL-17A, and IL-10 expression as described previously (39).

$16 S$ rRNA amplicon sequencing and analysis. DNA was extracted from 50-100 mg of fecal material using the Maxwell 16 Tissue DNA Kit (Promega). The 16S rRNA gene encompassing the V6 to V8 regions was amplified and sequenced on the MiSeq Sequencing System (Illumina) using paired-end sequencing with V3 300 bp chemistry in the Australian Centre for Ecogenomics according to the manufacturer's protocol. OTUs were identified using the QIIME script pick_open reference_otus.py (79) with default parameters (97\% similarity) and assigned taxonomy using BLAST (80) against the Greengenes reference database version 2014/0955 (http://data.ace.uq.edu.au/ public/gg). Differential abundance analysis was performed on raw read counts using DESeq2. Full details are provided in the Supplemental Methods. Sequencing data have been uploaded to the Sequence Read Archive under BioProject PRJNA433452.

RNA-seq and analysis. Total RNA was isolated from proximal colon and distal ileum tissue using Precellys Lysing and QIAGEN RNeasy
Plus Mini Kits per the manufacturer's instructions. RNA libraries were prepared using the NEBnext Ultra RNA Library Prep Kit for Illumina (New England Biolabs), assessed for size, and quantified using the 2100 Bioanalyzer (Agilent Technologies) and Qubit Fluorometer (Thermo Fisher Scientific), respectively. Libraries were sequenced using high-output single-end 75 cycle sequencing kits (version 2) on the Illumina NextSeq 550 platform. Full details are provided in Supplemental Methods. Sequencing data have been uploaded into ArrayExpress, accession number E-MTAB-6547.

Statistics. Survival curves were plotted using Kaplan-Meier estimates and compared by log-rank analysis. The parametric unpaired $t$ test or the nonparametric Mann-Whitney $U$ or $t$ tests (2-sided) were used for the statistical analysis of data. $P<0.05$ was considered statistically significant. Data are presented as mean \pm SEM.

Study approval. All animal procedures were carried out with approval from the QIMR Berghofer Medical Research Institute Animal Ethics Committee.

\section{Author contributions}

$\mathrm{AV}$ and MDB designed and performed experiments, analyzed and interpreted data, and wrote the manuscript. MK designed and performed experiments and contributed to data interpretation and discussion. KLO and NL generated and/or analyzed microbiome data and reviewed the manuscript. SDO, RDK, RJR, $\mathrm{ASH}$, and LC performed experiments. KHG contributed to discussions. RDK, OL, LKN, JYWM, DPF, JM, and JR provided and/ or generated critical reagents and reviewed the manuscript. ADC performed blinded histological assessment. JS and SWL performed analysis and/or contributed to interpretation of RNA-seq data. $\mathrm{PH}$ designed experiments, analyzed and interpreted data, and reviewed the manuscript. GRH designed experiments, interpreted data, and wrote the manuscript.

\section{Acknowledgments}

This study was supported by an Australian National Health and Medical Research Council (NH\&MRC) program grant held by GRH and PH; and a Cancer Council Queensland project grant held by AV, PH, GRH and KLO. We are grateful to Futoshi Kawamata, Paul Collins, Stephen Kazakoff, and Nic Waddell at QIMR Berghofer Medical Research Institute for assistance with RNA-seq. We thank Kelly Locke, Derek Weinert, and Luke Samson at QIMR Berghofer Medical Research Institute for technical assistance. We gratefully acknowledge Nicola Angel and Serene Low from the Australian Centre for Ecogenomics for assistance with sample preparation and $16 \mathrm{~S}$ rRNA sequencing.

Address correspondence to: Geoffrey Hill or Antiopi Varelias, QIMR Berghofer Medical Research Institute, 300 Herston Road, Herston, QLD, Australia, 4006. Phone: 61.7.3845.3763; Email: geoff.hill@qimrberghofer.edu.au (G.Hill).Phone: 61.7.3362.0322; Email: antiopi.varelias@qimrberghofer.edu.au (A. Varelias).
1. Markey KA, MacDonald KP, Hill GR. The biology of graft-versus-host disease: experimental systems instructing clinical practice. Blood. 2014;124(3):354-362.

2. Koyama M, Hill GR. Alloantigen presentation and graft-versus-host disease: fuel for the fire. Blood. 2016;127(24):2963-2970.

3. Koyama M, et al. Donor colonic CD103+dendritic cells determine the severity of acute graft-versushost disease. J Exp Med. 2015;212(8):1303-1321.
4. Hill GR, Ferrara JL. The primacy of the gastrointestinal tract as a target organ of acute graftversus-host disease: rationale for the use of cytokine shields in allogeneic bone marrow transplantation. Blood. 2000;95(9):2754-2759. 
5. Cua DJ, Tato CM. Innate IL-17-producing cells: the sentinels of the immune system. Nat Rev Immunol. 2010;10(7):479-489.

6. Chen Z, et al. Mucosal-associated invariant T-cell activation and accumulation after in vivo infection depends on microbial riboflavin synthesis and co-stimulatory signals. Mucosal Immunol. 2017;10(1):58-68.

7. Rahimpour A, et al. Identification of phenotypically and functionally heterogeneous mouse mucosal-associated invariant $\mathrm{T}$ cells using MR1 tetramers. JExp Med. 2015;212(7):1095-1108.

8. Reantragoon R, et al. Antigen-loaded MR1 tetramers define $\mathrm{T}$ cell receptor heterogeneity in mucosal-associated invariant $\mathrm{T}$ cells. J Exp Med. 2013;210(11):2305-2320.

9. Kawachi I, Maldonado J, Strader C, Gilfillan S. MR1-restricted V alpha 19i mucosal-associated invariant $\mathrm{T}$ cells are innate $\mathrm{T}$ cells in the gut lamina propria that provide a rapid and diverse cytokine response. JImmunol. 2006;176(3):1618-1627.

10. Godfrey DI, Uldrich AP, McCluskey J, Rossjohn J, Moody DB. The burgeoning family of unconventional T cells. Nat Immunol. 2015;16(11):1114-1123.

11. Corbett AJ, et al. T-cell activation by transitory neo-antigens derived from distinct microbial pathways. Nature. 2014;509(7500):361-365.

12. Eckle SB, et al. Recognition of vitamin B precursors and byproducts by mucosal associated invariant T cells. J Biol Chem. 2015;290(51):30204-30211.

13. Kjer-Nielsen L, et al. MR1 presents microbial vitamin B metabolites to MAIT cells. Nature. 2012;491(7426):717-723.

14. Patel O, et al. Recognition of vitamin B metabolites by mucosal-associated invariant $\mathrm{T}$ cells. $\mathrm{Nat}$ Commun. 2013;4:2142.

15. Okada $S$, et al. Immunodeficiencies. Impairment of immunity to Candida and Mycobacterium in humans with bi-allelic RORC mutations. Science. 2015;349(6248):606-613.

16. Gold MC, Lewinsohn DM. Co-dependents: MR1-restricted MAIT cells and their antimicrobial function. Nat Rev Microbiol. 2013;11(1):14-19.

17. Georgel P, Radosavljevic M, Macquin C, Bahram $\mathrm{S}$. The non-conventional MHC class I MR1 molecule controls infection by Klebsiella pneumoniae in mice. Mol Immunol. 2011;48(5):769-775.

18. Le Bourhis L, et al. Antimicrobial activity of mucosal-associated invariant T cells. Nat Immunol. 2010;11(8):701-708.

19. Chua WJ, Truscott SM, Eickhoff CS, Blazevic A, Hoft DF, Hansen TH. Polyclonal mucosaassociated invariant $\mathrm{T}$ cells have unique innate functions in bacterial infection. Infect Immun . 2012;80(9):3256-3267.

20. Dusseaux M, et al. Human MAIT cells are xenobiotic-resistant, tissue-targeted, CD161hi IL-17secreting T cells. Blood. 2011;117(4):1250-1259.

21. Cui Y, et al. Mucosal-associated invariant T cellrich congenic mouse strain allows functional evaluation. J Clin Invest. 2015;125(11):4171-4185.

22. Meierovics A, Yankelevich WJ, Cowley SC. MAIT cells are critical for optimal mucosal immune responses during in vivo pulmonary bacterial infection. Proc Natl Acad Sci U S A. 2013;110(33):E3119-E3128.

23. Le Bourhis L, Mburu YK, Lantz O. MAIT cells, surveyors of a new class of antigen: development and functions. Curr Opin Immunol. 2013;25(2):174-180.

24. Godfrey DI, Rossjohn J, McCluskey J. Fighting infection with your MAITs. Nat Immunol. 2010;11(8):693-695.

25. Croxford JL, Miyake S, Huang YY, Shimamura M, Yamamura T. Invariant $\mathrm{V}$ (alpha)19i $\mathrm{T}$ cells regulate autoimmune inflammation. Nat Immunol. 2006;7(9):987-994.

26. Chiba A, Tajima R, Tomi C, Miyazaki Y, Yamamura T, Miyake S. Mucosal-associated invariant $\mathrm{T}$ cells promote inflammation and exacerbate disease in murine models of arthritis. Arthritis Rheum. 2012;64(1):153-161.

27. Hinks TS. Mucosal-associated invariant T cells in autoimmunity, immune-mediated diseases and airways disease. Immunology. 2016;148(1):1-12.

28. Treiner E. Mucosal-associated invariant $T$ cells in inflammatory bowel diseases: bystanders, defenders, or offenders? Front Immunol. 2015;6:27.

29. Barnes MJ, Powrie F. Regulatory T cells reinforce intestinal homeostasis. Immunity. 2009;31(3):401-411.

30. Atarashi K, et al. Induction of colonic regulatory $\mathrm{T}$ cells by indigenous Clostridium species. Science. 2011;331(6015):337-341.

31. Atarashi $\mathrm{K}$, et al. Treg induction by a rationally selected mixture of Clostridia strains from the human microbiota. Nature. 2013;500 (7461):232-236.

32. Arpaia N, et al. Metabolites produced by commensal bacteria promote peripheral regulatory $\mathrm{T}$-cell generation. Nature. 2013;504(7480):451-455.

33. Furusawa Y, et al. Commensal microbe-derived butyrate induces the differentiation of colonic regulatory T cells. Nature. 2013;504(7480):446-450.

34. Shimamura M, Huang YY, Kobayashi M, Goji H. Altered production of immunoregulatory cytokines by invariant Valpha19 TCR-bearing cells dependent on the duration and intensity of TCR engagement. Int Immunol. 2009;21(2):179-185.

35. Hanash AM, et al. Interleukin-22 protects intestinal stem cells from immune-mediated tissue damage and regulates sensitivity to graft versus host disease. Immunity. 2012;37(2):339-350.

36. Mathewson ND, et al. Gut microbiome-derived metabolites modulate intestinal epithelial cell damage and mitigate graft-versus-host disease. Nat Immunol. 2016;17(5):505-513.

37. Kumar $P$, et al. Intestinal interleukin-17 receptor signaling mediates reciprocal control of the gut microbiota and autoimmune inflammation. Immunity. 2016;44(3):659-671.

38. O'Connor $\mathrm{W}$, et al. A protective function for interleukin 17A in T cell-mediated intestinal inflammation. Nat Immunol. 2009;10(6):603-609.

39. Varelias A, et al. Acute graft-versus-host disease is regulated by an IL-17-sensitive microbiome. Blood. 2017;129(15):2172-2185.

40. O'Connor BP, et al. Semaphorin 6D regulates the late phase of $\mathrm{CD} 4+\mathrm{T}$ cell primary immune responses. Proc Natl Acad Sci U S A. 2008;105(35):13015-13020.

41. Nakagawa $Y$, et al. Identification of semaphorin $4 \mathrm{~B}$ as a negative regulator of basophil-mediated immune responses. J Immunol.

2011;186(5):2881-2888
42. Geiger TL, et al. Nfil3 is crucial for development of innate lymphoid cells and host protection against intestinal pathogens. J Exp Med. 2014;211(9):1723-1731.

43. Wu B, Kulkarni K, Basu S, Zhang S, Hu M. First-pass metabolism via UDP-glucuronosyltransferase: a barrier to oral bioavailability of phenolics. JPharm Sci. 2011;100(9):3655-3681.

44. Godfrey DI, Rossjohn J, McCluskey J. Fighting infection with your MAITs. Nat Immunol. 2010;11(8):693-695.

45. Ussher JE, et al. CD161++ CD8+ T cells, including the MAIT cell subset, are specifically activated by IL-12+IL-18 in a TCR-independent manner. Eur J Immunol. 2014;44(1):195-203.

46. Loh L, et al. Human mucosal-associated invariant $\mathrm{T}$ cells contribute to antiviral influenza immunity via IL-18-dependent activation. Proc Natl Acad Sci U S A. 2016;113(36):10133-10138.

47. van Wilgenburg B, et al. MAIT cells are activated during human viral infections. Nat Commun. 2016;7:11653.

48. Ormerod KL, et al. Genomic characterization of the uncultured Bacteroidales family S24-7 inhabiting the guts of homeothermic animals. Microbiome. 2016;4(1):36.

49. Bettelli E, Korn T, Oukka M, Kuchroo VK. Induction and effector functions of $\mathrm{T}(\mathrm{H}) 17$ cells. Nature. 2008;453(7198):1051-1057.

50. Fragoulis GE, Siebert S, McInnes IB. Therapeutic Targeting of IL-17 and IL-23 Cytokines in Immune-Mediated Diseases. Annu Rev Med. 2016;67:337-353.

51. Chong HJ, et al. IL-4 selectively enhances FcgammaRIII expression and signaling on mouse mast cells. Cell Immunol. 2003;224(2):65-73.

52. Takahashi N, et al. IL-17 produced by Paneth cells drives TNF-induced shock. JExp Med. 2008;205(8):1755-1761.

53. Liang SC, et al. Interleukin (IL)-22 and IL-17 are coexpressed by Th17 cells and cooperatively enhance expression of antimicrobial peptides. JExp Med.2006;203(10):2271-2279.

54. Song X, et al. Growth factor FGF2 cooperates with interleukin-17 to repair intestinal epithelial damage. Immunity. 2015;43(3):488-501.

55. Lee JS, et al. Interleukin-23-independent IL-17 production regulates intestinal epithelial permeability. Immunity. 2015;43(4):727-738.

56. Bär E, Whitney PG, Moor K, Reis e Sousa C, LeibundGut-Landmann S. IL-17 regulates systemic fungal immunity by controlling the functional competence of NK cells. Immunity. 2014;40(1):117-127.

57. Huang W, Na L, Fidel PL, Schwarzenberger P. Requirement of interleukin-17A for systemic anti-Candida albicans host defense in mice. J Infect Dis. 2004;190(3):624-631.

58. Ishigame $\mathrm{H}$, et al. Differential roles of interleukin$17 \mathrm{~A}$ and $-17 \mathrm{~F}$ in host defense against mucoepithelial bacterial infection and allergic responses. Immunity. 2009;30(1):108-119.

59. Ouyang W, Kolls JK, Zheng Y. The biological functions of $\mathrm{T}$ helper 17 cell effector cytokines in inflammation. Immunity. 2008;28(4):454-467.

60. Raffatellu M, et al. Simian immunodeficiency virus-induced mucosal interleukin-17 deficiency promotes Salmonella dissemination from the 
gut. Nat Med. 2008;14(4):421-428.

61. Gartlan $\mathrm{KH}$, et al. Tc17 cells are a proinflammatory, plastic lineage of pathogenic CD8+ T cells that induce GVHD without antileukemic effects. Blood. 2015;126(13):1609-1620.

62. Kappel LW, et al. IL-17 contributes to CD4mediated graft-versus-host disease. Blood. 2009;113(4):945-952.

63. Novak J, Dobrovolny J, Brozova J, Novakova L, Kozak T. Recovery of mucosal-associated invariant $\mathrm{T}$ cells after myeloablative chemotherapy and autologous peripheral blood stem cell transplantation. Clin Exp Med. 2016;16(4):529-537.

64. Hill GR, Ferrara JL. The primacy of the gastrointestinal tract as a target organ of acute graft-versus-host disease: rationale for the use of cytokine shields in allogeneic bone marrow transplantation. Blood. 2000;95(9):2754-2759.

65. Seach N, et al. Double-positive thymocytes select mucosal-associated invariant T cells. J Immunol. 2013;191(12):6002-6009.

66. Bhattacharyya A, et al. Graft-derived reconstitution of mucosal-associated invariant $\mathrm{T}$ cells after allogeneic hematopoietic cell transplantation. Biol Blood Marrow Transplant. 2018;24(2):242-251.
67. Treiner E, et al. Selection of evolutionarily conserved mucosal-associated invariant $\mathrm{T}$ cells by MR1. Nature. 2003;422(6928):164-169.

68. Martin E, et al. Stepwise development of MAIT cells in mouse and human. PLoS Biol. 2009;7(3):e54.

69. Lantz O, Grandjean I, Matzinger P, Di Santo JP. Gamma chain required for naïve CD4+ T cell survival but not for antigen proliferation. Nat Immunol. 2000;1(1):54-58.

70. Beilhack A, et al. In vivo analyses of early events in acute graft-versus-host disease revea sequential infiltration of T-cell subsets. Blood. 2005;106(3):1113-1122.

71. Tittel AP, et al. Functionally relevant neutrophilia in CD11c diphtheria toxin receptor transgenic mice. Nat Methods. 2012;9(4):385-390.

72. Hirota K, et al. Fate mapping of IL-17-producing $\mathrm{T}$ cells in inflammatory responses. Nat Immunol. 2011;12(3):255-263.

73. Creusot RJ, et al. Lymphoid-tissue-specific homing of bone-marrow-derived dendritic cells. Blood. 2009;113(26):6638-6647.

74. Cooke KR, et al. An experimental model of idiopathic pneumonia syndrome after bone marrow transplantation: I. The roles of minor $\mathrm{H}$ antigens and endotoxin. Blood. 1996;88(8):3230-3239.

75. Sakala IG, et al. Functional heterogeneity and antimycobacterial effects of mouse mucosalassociated invariant $\mathrm{T}$ cells specific for riboflavin metabolites. JImmunol. 2015;195(2):587-601.

76. Varelias A, et al. Lung parenchyma-derived IL-6 promotes IL-17A-dependent acute lung injury after allogeneic stem cell transplantation. Blood. 2015;125(15):2435-2444.

77. Kuns RD, et al. Invariant natural killer $\mathrm{T}$ cell-natural killer cell interactions dictate transplantation outcome after alphagalactosylceramide administration. Blood. 2009;113(23):5999-6010.

78. Mak JY, et al. Stabilizing short-lived Schiff base derivatives of 5-aminouracils that activate mucosal-associated invariant T cells. Nat Commun. 2017;8:14599.

79. Caporaso JG, et al. QIIME allows analysis of high-throughput community sequencing data. Nat Methods. 2010;7(5):335-336.

80. Altschul SF, Gish W, Miller W, Myers EW, Lipman DJ. Basic local alignment search tool. JMol Biol. 1990;215(3):403-410. 